\title{
Does visuomotor adaptation contribute to illusion-resistant grasping?
}

\author{
Evan Cesanek $^{1}$ - Carlo Campagnoli ${ }^{2} \cdot$ Jordan A. Taylor $^{2} \cdot$ Fulvio Domini $^{1,3}$
}

Published online: 29 August 2017

(C) Psychonomic Society, Inc. 2017

\begin{abstract}
Do illusory distortions of perceived object size influence how wide the hand is opened during a grasping movement? Many studies on this question have reported illusion-resistant grasping, but this finding has been contradicted by other studies showing that grasping movements and perceptual judgments are equally susceptible. One largely unexplored explanation for these contradictions is that illusion effects on grasping can be reduced with repeated movements. Using a visuomotor adaptation paradigm, we investigated whether an adaptation model could predict the time course of Ponzo illusion effects on grasping. Participants performed a series of trials in which they viewed a thin wooden target, manually reported an estimate of the target's length, then reached to grasp the target. Manual size estimates (MSEs) were clearly biased by the illusion, but maximum grip apertures (MGAs) of grasping movements were consistently accurate. Illusion-resistant MGAs were observed immediately upon presentation of the illusion, so there was no decrement in susceptibility for the adaptation model to explain. To determine whether online corrections based on visual feedback could have produced illusion-resistant MGAs, we performed an exploratory post hoc analysis of movement trajectories. Early portions of the illusion effect profile evolved as if they were biased by the illusion to the same magnitude as the perceptual responses (MSEs), but this bias was attenuated prior to the MGA. Overall, this preregistered
\end{abstract}

Fulvio Domini

Fulvio_Domini@brown.edu

1 Brown University, Box 1821, 190 Thayer Street, Providence, RI 02912, USA

2 Princeton University, Princeton, NJ, USA

3 Center for Neuroscience and Cognitive Systems@UniTn, Rovereto, Italy study demonstrated that visuomotor adaptation of grasping is not the primary source of illusion resistance in closed-loop grasping.

Keywords Reach-to-grasp · Manual size estimation · Ponzo illusion $\cdot$ Visuomotor adaptation

\section{Introduction}

\section{Are two visual systems needed to explain differences between perception and action?}

In the two visual streams hypothesis (TVSH; Goodale \& Milner, 1992; Milner \& Goodale, 2008), the broad anatomical distinction between the ventral and dorsal streams of the human visual system is given an appealing functional interpretation: the ventral stream produces conscious visual perception whereas the dorsal stream allows visually guided action, and each performs visual processing that is different in style and independent of processing in the other stream. In particular, the ventral vision-for-perception stream is said to rely on a relative encoding scheme (e.g., bigger/ smaller, nearer/farther) to support stable percepts, while the dorsal vision-for-action stream is said to rapidly compute the absolute location and 3-D shapes of target objects in an online fashion and ignore scene context to enable accurate and efficient movements (Dyde \& Milner, 2002; Goodale, Gonzalez, \& Kroliczak, 2008). These proposed differences are the basis for the prediction that motor actions will not be tricked by context-induced distortions of perceived object properties.

The primary alternative to the TVSH is the hypothesis that unitary visual estimates of object properties, encoded in a number of regions throughout the visual brain, drive perceptual and motoric responses as needed (Smeets \& Brenner, 1999; Franz, Fahle, Bülthoff, \& Gegenfurtner, 2001). This common representation hypothesis is often associated with the prediction that 
motor actions will be influenced by context-induced distortions to the same extent as conscious perceptual reports (assuming that both responses are driven by the same visual feature; cf. Brenner $\&$ Smeets, 1996). However, as we will show, the common representation hypothesis is not bound to this prediction, as there are other ways that grasping movements can exceed the accuracy of apparently well-matched perceptual responses.

\section{Illusions and grasping}

The debate over whether visual illusions influence grasping was ignited by Aglioti, DeSouza, and Goodale's (1995) widely cited study using the Ebbinghaus illusion, which found a smaller illusion effect on grasping $(1.6 \mathrm{~mm})$ than on perceptual matching $(2.5 \mathrm{~mm})$. Since then, studies have focused on ensuring proper matching of perceptual and grasping tasks to avoid enhanced or reduced effects in either response that are not attributable to the visual size estimate. Briefly, proper matching includes (1) equivalent availability of online visual information (Post \& Welch, 1996), (2) equivalent attention to display elements (Franz, Gegenfurtner, Bülthoff, \& Fahle, 2000; Pavani, Boscagli, Benvenuti, Rabuffetti, \& Farnè, 1999), (3) equivalent haptic feedback (Haffenden \& Goodale, 1998; Vishton, Rea, Cutting, \& Nuñez, 1999), (4) the absence of obstacle avoidance and other confounding motor effects caused by illusory display elements but not due to distortions of perceived size (Haffenden \& Goodale, 2000; Haffenden, Schiff, \& Goodale, 2001), and (5) accounting for differences in the slopes of response functions (Franz et al., 2001). Notably, Franz and colleagues have demonstrated that when all of these factors are accounted for, the data from multiple studies using the Ebbinghaus illusion show consistently comparable illusion effects on grasping and perception, lending support to the common representation hypothesis (Franz \& Gegenfurtner, 2008; Kopiske, Bruno, Hesse, Schenk, \& Franz, 2016).

Using other illusions, however, multiple studies have shown illusion-resistant grasping despite controlling for the factors mentioned above (Ganel, Tanzer, \& Goodale, 2008; Stöttinger, Aigner, Hanstein, \& Perner, 2009; Stöttinger, Soder, Pfusterschmied, Wagner, \& Perner, 2010). Thus, there are at least two possible interpretations of the mixed evidence: (1) the TVSH proponent might argue that vision-for-action is immune to some, but not all, context-induced visual distortions, whereas (2) the common representation proponent might argue that additional unaccounted-for factors can explain reduced illusion effects on grasping found in otherwise well-controlled studies. In this study, we sought to evaluate whether visuomotor adaptation could be one such additional factor.

\section{Adapting grasping movements to the Ponzo illusion}

The motivation for this study was the observation that the sensorimotor system makes trial-by-trial adjustments to grasp coordination based on sensory feedback registered during each movement (Bingham, Coats, \& Mon-Williams, 2007; Säfström \& Edin, 2008; Schenk, 2012). This implies that the session-wise averages of multiple grasps reported in most previous studies do not provide a pure measure of the underlying visual size estimate. Within-session decrements have been found previously in the effects of (1) the Ponzo illusion on MGAs (Gonzalez, Ganel, Whitwell, Morrissey, \& Goodale, 2008; Whitwell, Buckingham, Enns, Chouinard, \& Goodale, 2016), (2) an orientation illusion on grip orientation (Glover \& Dixon, 2001b), and (3) the size-weight illusion on grip forces (Flanagan \& Beltzner, 2000; Grandy \& Westwood, 2006), providing preliminary support for the adaptation hypothesis. The present study extends previous work by (1) testing for trial-by-trial error corrections, (2) measuring the correlation between illusion effects on grasping and perception, and (3) applying a computational model of adaptation to quantify error correction processes.

This study used the Ponzo illusion (see Fig. 1), which may seem an odd choice given that several previous studies have concluded that maximum grip apertures (MGAs) are not affected by the illusion (Brenner \& Smeets, 1996; Bartelt \& Darling, 2002; Ganel et al., 2008; Gonzalez et al., 2008; Jackson \& Shaw, 2000). However, recent evidence contradicts these findings: Whitwell et al. (2016) found that closed-loop grasps occurring early in the trial sequence showed an illusion effect comparable to manual size estimates, a trend that we too observed in a pilot study (see below). We aimed to address this uncertainty in the present study by recruiting a large sample and utilizing a four-phase adaptation design in order to monitor temporal changes. Whitwell et al.'s (2016) findings and our pilot data also supported the assumption that visuomotor adaptation would not affect our perceptual measure, manual size estimates (MSEs). Though not a traditional perceptual measure, MSEs are useful when comparing perception with grasping because, like MGAs, they are continuous and involve the precision grip as the end effector (Haffenden \& Goodale, 1998). While it might be rightly argued that MSEs are, by virtue of being hand movements, quasimotoric responses and therefore potentially susceptible to adaptation, previous findings have supported the view that MSEs are read-outs of perceived size, similar to adjustable probes.

If the Ponzo illusion does affect grasping, as suggested by the common representation model, the adaptation hypothesis makes the additional prediction that illusion effects on MGAs can be reduced with practice via adaptation of the mappings that transform visual size information into grasp control parameters. To formalize the adaptation hypothesis, we implemented a trial-by-trial error correction process with a linear state-space model (Cheng \& Sabes, 2006; Thoroughman \& Shadmehr, 2000). First, our model assumes that on each trial the visual system computes an estimate of target size that may differ from the physical target size. Next, we model grasp 


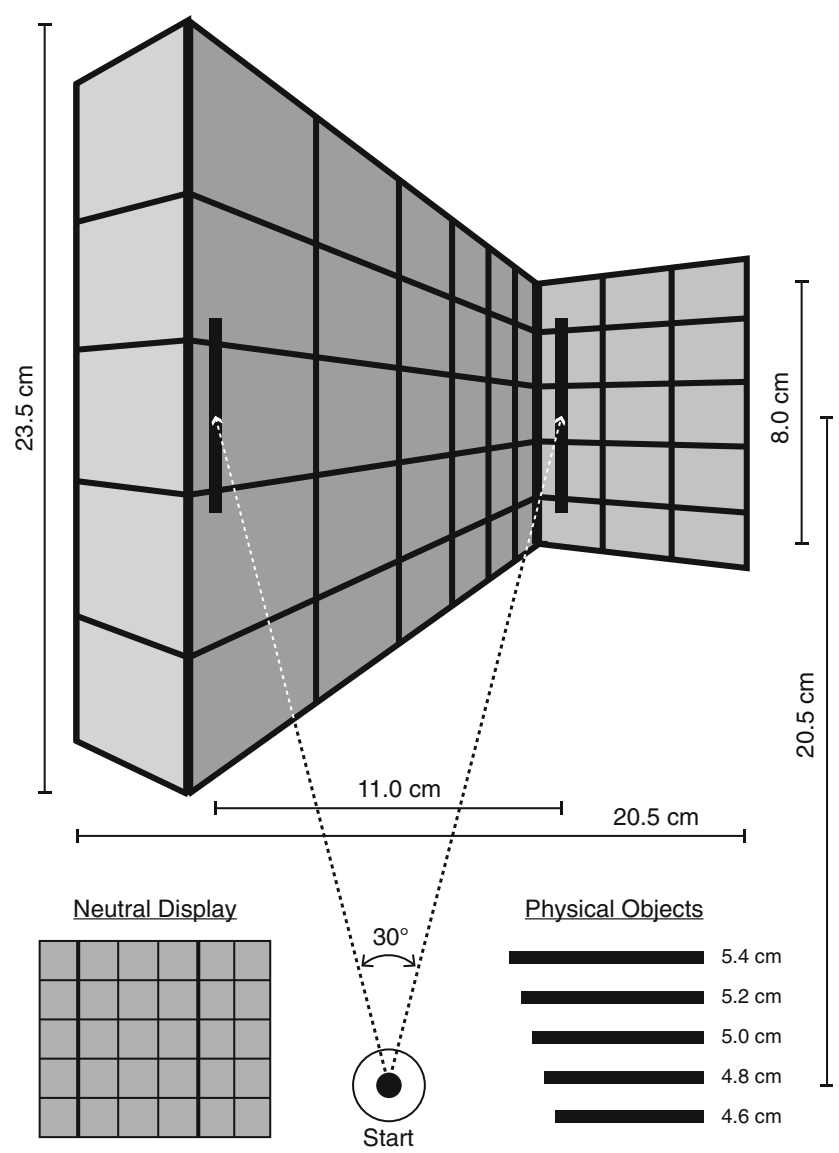

Fig. 1 Geometry of the Ponzo illusion display and the starting hand position. Illusion is depicted with two physically equal-size objects to demonstrate perceived size distortion, but experimental trials included only one object. At bottom left, neutral grid used during baseline and washout phases. At bottom right, all five physical objects used during baseline and washout; only the middle three sizes were used during the illusion-adaptation phase. The perceptually equalized phase involved two pairs of physical objects chosen from a larger set that were estimated from each participant's slope-corrected MSE effect

planning with a linear response function that maps perceived size onto MGA; this is likely a simplification of the actual grasp planning process, but it will suffice because the MGA effectively summarizes changes in grasp execution across different object sizes (Jeannerod, 1984). The response function involves fixed slope and intercept parameters that are specific to each individual, plus an additional offset parameter modeled as a dynamic internal state that can be adjusted in response to detected movement errors. Specific properties of error signals in grasp adaptation are not well understood, so we assumed only that these signals indicate by how much the grasp was too large or too small.

For the purposes of fitting the model, this error signal was approximated quantitatively by comparing the observed MGA on each trial to an "ideal MGA," estimated based on each participant's grasping performance over many trials. This comparison operationalized the idea that the system interprets sensory feedback from an inaccurate movement as a deviation from an idealized movement pattern (e.g., the signals could indicate a lack of smoothness, as in popular trajectory optimization models; Flash \& Hogan, 1985). We distinguish this from the notion that feedback provides information directly related to the size of the target. After each movement, the error feedback signal adjusts the visuomotor mapping according to an update rule that (1) decays the internal state and (2) extracts from it some of the detected movement error.

Since most illusion and grasping studies involve randomly interleaved presentations of size-increasing and sizedecreasing contexts, the visuomotor mapping must be adjusted positively for one context and negatively (or not at all) for the other in order to acquire illusion resistance. The adaptation literature suggests that this is indeed possible under the right circumstances: When a movement error is detected, the error signal primarily influences subsequent movements that are "contextually similar"; for dissimilar movements, the error signal has a reduced corrective influence (Krakauer, Ghez, \& Ghilardi, 2005). The features that constitute contextual similarity in visuomotor adaptation are not fully understood, but spatial/angular separation between movement endpoints has been identified as a key factor in reaching experiments (Ghahramani, Wolpert, \& Jordan, 1996; Pine, Krakauer, Gordon, \& Ghez, 1996). Therefore, consistently presenting the size-increasing and size-decreasing contexts in separate spatial locations may allow simultaneous compensation for these opposing size distortions. Conversely, if conflicting error signals are experienced in a single location, the visuomotor mapping may oscillate between compensatory states, preventing successful adaptation to the illusion. At the same time, we cannot rule out the possibility that adaptation occurred in studies using a single target location, as other distinguishing visual and/or kinematic features may have been available. As described in the next section, the present study was also designed to test for this proposed effect of spatial separation.

\section{Predictions}

The state-space model produced a number of testable predictions; preliminary support for many of these was found in the pilot data. First, if illusion resistance in grasp control is acquired via visuomotor adaptation, the MGA should initially reflect illusion-distorted object sizes but gradually become tuned to physical sizes, whereas MSEs should remain consistently biased. On the other hand, if MGAs remain consistently biased, this would suggest (1) that errors are not being detected or (2) that error corrections of opposite sign are canceling out (i.e., full interference). Second, if a trial-by-trial error correction process produces a decrement in the illusion effect on action, we should observe a previous-trial effect on the MGA in early grasps, where grasps following contact with an illusory-small object are made larger, and grasps following 
contact with an illusory-large object are made smaller. Third, applying the model should confirm the adaptation hypothesis by estimating nonzero error correction and generalization parameters, as well as a significant illusion effect. Lastly, if a common visual size estimate drives both perception and grasping, early illusion effects on MGA and MSE should be correlated. One limitation of this study is that if MGAs are found to be illusion-resistant even in early trials, then we may not be able to conclusively determine whether illusion resistance is due to veridical vision-for-action or other accounts that do not require accurate vision-for-action (e.g., positionbased grasp planning, Smeets \& Brenner, 1999, or closedloop online control, Franz, Hesse, \& Kollath, 2009).

At the end of the experiment, we included a short block of trials where two pairs of perceptually equalized target objects (selected based on each participant's slope-corrected MSE effect during the illusion-adaptation phase) were presented in a single target location, alternating every trial between the illusory-large and illusory-small contexts. The visuomotor system should not be able to maintain separate states for the two illusion contexts under these conditions if this function depends on consistent spatial separation. In fact, the error correction model predicts a slight enhancement of the illusion effect on grasping because the trial-by-trial corrections, which depend on the previous trial type, are always in the same direction as the expected illusion effect on the current trial. An illusion effect that is larger than the effect in the illusionadaptation phase would provide additional support for the proposed model, while a decrement in the illusion effect in this phase would suggest that spatial separation is not necessary to adapt to the illusion.

\section{Method}

\section{Participants}

To determine the required number of participants, we performed power analyses on each of our three main predictions (alpha-level $=0.05$; desired power $=0.98$ ). (1) Based on the published means and $t$ values for Whitwell et al.'s (2016) decrement in the illusion effect from the first five to the last five bins, we estimated an effect size of $d=.426$, which would require 77 participants to reliably detect in a one-tailed pairedsamples $t$ test. In contrast, the effect size estimated from our pilot data for the change in illusion effect from Bin 2 (maximal value) to Bin 5 (minimal value) was $d=.255$, which would require 212 participants, but we suspected that our collected data would be less noisy due to constrained trial sequencing, the use of a single infrared-emitting diode (IRED), and the baseline phase allowing participants to establish a consistent grasp strategy before the illusion was introduced. (2) We used the pilot data to estimate an effect size of $d=.502$ for the previous-trial effect on MGAs, which would require 56 participants to detect with a one-tailed paired-samples $t$ test. (3) We used the pilot data to estimate an effect size of $r=.405$ for the MGA-MSE correlation in the first trial bin, which would require 72 participants to reliably detect. We also computed Bayes factors for specific tests as follows: The null hypothesis prediction ( $\mathrm{H} 0)$ was always a point distribution and the alternative hypothesis predictions (H1) were various normal and uniform distributions specified according to the observed effects of our Ponzo display combined with the adaptation model predictions (see Table 1).

Based on the power analyses, we planned to recruit 80 participants from Brown University for participation in this study. We used a multiple of four because we aimed to balance the number of participants in each of our four groups (two trial-sequence groups crossed with two illusion-orientation groups). Prospective participants were required to selfidentify as right-handed with normal or corrected-to-normal vision in order to participate in the study. Participants were asked to give informed consent prior to any participation and granted course credit or paid $\$ 8$ per hour as compensation, following a protocol approved by the Brown University Institutional Review Board.

\section{Materials}

The target objects were thin wooden dowels (4.9 $\mathrm{mm}$ wide $\times$ $3.2 \mathrm{~mm}$ deep) cut to 17 different lengths ( 46.0 to $54.0 \mathrm{~mm}$ in 0.5 -mm increments) and painted matte black. The illusionadaptation phase involved only the 48-, 50-, and 52-mm targets; the 46- and 54-mm targets were presented during baseline and washout to prevent participants from memorizing a limited set of target sizes prior to the illusion-adaptation phase. The rest of the targets were used when selecting two pairs of perceptually equalized targets for the perceptually equalized phase. To select each pair, we found two objects that differed by the participant's average slope-corrected MSE effect during illusion-adaptation, rounded to the nearest integer in order to accommodate our 0.5 $\mathrm{mm}$ resolution. The two pairs were centered on 48 and $52 \mathrm{~mm}$; slope-corrected MSE effects greater than $4 \mathrm{~mm}$ were rounded down to $4 \mathrm{~mm}$ for the purposes of the perceptual match.

Each stimulus display contained one target object placed on the left or right side of the current display. The displays were mounted on a $22 \times 28 \times 1 \mathrm{~cm}$ piece of black foam board, which was magnetically affixed to a flat black tabletop. The left-right orientation of the two illusory contexts was counterbalanced across participants. In the main experimental series, the distance between the two target positions was 11 $\mathrm{cm}$. In the perceptually equalized phase, all targets were presented along the participant's midline and the display was shifted laterally on each trial to alternate the illusory contexts. In all trials, the start position was a point near the edge of the tabletop, located $20.5 \mathrm{~cm}$ proximally from the center of the 
Table 1 Specification of prior distributions for Bayes factors

\begin{tabular}{|c|c|c|c|}
\hline Preregistered test & & $\mathrm{H} 0$ & $\mathrm{H} 1$ \\
\hline $\begin{array}{l}\text { Illusion effect on MGA in Bin } 1 \\
\mathrm{BF}=0.01\end{array}$ & $\begin{array}{l}\text { Theory } \\
\text { Distribution } \\
\text { Parameters }\end{array}$ & $\begin{array}{l}\text { Two visual streams } \\
\text { Point } \\
\mu=0\end{array}$ & $\begin{array}{l}\text { Common representation } \\
\text { Normal } \\
\mu=\text { mean of corrected MSE effect in Bin } 1 \\
\sigma=S E M \text { of corrected MSE effect in Bin } 1\end{array}$ \\
\hline $\begin{array}{l}\text { MGA } \times \text { MSE illusion effect correlation } \\
\text { in Bins } 1 \& 2 \\
\mathrm{BF}=3.75\end{array}$ & $\begin{array}{l}\text { Theory } \\
\text { Distribution } \\
\text { Parameters }\end{array}$ & $\begin{array}{l}\text { Two visual streams } \\
\text { Point } \\
\mu=0\end{array}$ & $\begin{array}{l}\text { Common representation } \\
\text { Uniform } \\
\text { Lower bound }=0 \\
\text { Upper bound }=\text { Fisher } z \text {-transformed maximal expected } \\
\text { correlation* }\end{array}$ \\
\hline $\begin{array}{l}\text { Effect on MGA vs. effect on MSE during } \\
\text { illusion-adaptation }\end{array}$ & Theory & $\begin{array}{l}\text { Common } \\
\text { representation }\end{array}$ & Two visual streams \\
\hline $\mathrm{BF}>10000$ & $\begin{array}{l}\text { Distribution } \\
\text { Parameters }\end{array}$ & $\begin{array}{l}\text { Point } \\
\mu=0\end{array}$ & $\begin{array}{l}\text { Normal } \\
\mu=\text { mean of corrected MSE effect during illusion-adaptation } \\
\sigma=S E M \text { of corrected MSE effect during illusion-adaptation }\end{array}$ \\
\hline $\begin{array}{l}\text { Decrement in effect on MGA from } \\
\text { Bin } 1 \text { to Bin } 6 \\
\mathrm{BF}=3.55\end{array}$ & $\begin{array}{l}\text { Theory } \\
\text { Distribution } \\
\text { Parameters }\end{array}$ & $\begin{array}{l}\text { No adaptation } \\
\text { Point } \\
\mu=0\end{array}$ & $\begin{array}{l}\text { Adaptation } \\
\text { Normal } \\
\mu=79.1 \% \text { of MGA effect in Bin } 1 * * \\
\sigma=S E M \text { of } 79.1 \% \text { of MGA effect in Bin } 1\end{array}$ \\
\hline $\begin{array}{l}\text { Increased effect on MGA during } \\
\text { perceptually } \\
\text { equalized compared to during } \\
\text { illusion-adaptation } \\
\mathrm{BF}=0.10\end{array}$ & $\begin{array}{l}\text { Theory } \\
\text { Distribution } \\
\text { Parameters }\end{array}$ & $\begin{array}{l}\text { No adaptation } \\
\text { Point } \\
\mu=0\end{array}$ & $\begin{array}{l}\text { Adaptation } \\
\text { Normal } \\
\mu=422 \% \text { of MGA effect during illusion-adaptation } * * * \\
\sigma=S E M \text { of MGA effect during illusion-adaptation }\end{array}$ \\
\hline $\begin{array}{l}\text { Effect on MGA vs. effect on MSE during } \\
\text { perceptually } \\
\text { equalized }\end{array}$ & $\begin{array}{l}\text { Theory } \\
\text { Distribution }\end{array}$ & $\begin{array}{l}\text { Common } \\
\text { representation } \\
\text { Point }\end{array}$ & Two visual streams \\
\hline $\mathrm{BF}>1000$ & Parameters & $\mu=0$ & $\begin{array}{l}\mu=\text { mean of corrected MSE effect during perceptually equalized } \\
\sigma=S E M \text { of corrected MSE effect during perceptually equalized }\end{array}$ \\
\hline $\begin{array}{l}\text { Previous-trial effect on MGA in Bin } 1 \\
\mathrm{BF}=15.15\end{array}$ & $\begin{array}{l}\text { Theory } \\
\text { Distribution } \\
\text { Parameters }\end{array}$ & $\begin{array}{l}\text { No adaptation } \\
\text { Point } \\
\mu=0\end{array}$ & $\begin{array}{l}\text { Adaptation } \\
\text { Normal } \\
\mu=\text { revised adaptation model prediction based on MSE effect in } \\
\quad \text { illusion-adaptation } \\
\sigma=0.5 \times \mu * * * *\end{array}$ \\
\hline
\end{tabular}

\footnotetext{
*Maximal expected correlation computed by pooling Spearman-Brown split-half reliabilities on the MSE and MGA illusion effects in Bins 1, 3, and 5 versus Bins 2, 4, and 6 (Kopiske et al., 2016)

**Revised model simulations based on observed MSE effect in illusion-adaptation predicted a $79.1 \%$ decrement

****Model simulations predicted that the perceptually equalized phase would produce a mean illusion effect on MGA that was $422 \%$ of the mean illusion effect on MGA found during illusion-adaptation

****Half of model-predicted mean was used as standard deviation based on a suggestion by Dienes (2011)
}

display. This layout created an angular separation of 30 degrees between the two straight-line trajectories connecting the start position to the left and right target positions. A single IRED was applied to the thumbnail and another to the index fingernail of each participant. These IREDs were recorded at $85 \mathrm{~Hz}$ throughout each experiment using an Optotrak Certus (Northern Digital Inc.). IRED wires were kept away from the hand by applying Velcro straps to the wrist and forearm and a small piece of tape to the back of the hand. Liquid-crystal shutter glasses (PLATO goggles; Translucent Technologies) were used to control stimulus-viewing duration.

\section{Procedure}

\section{Trial procedure}

Participants were seated in a height-adjustable chair with the head unconstrained. At the start of each trial, a single beep was played aloud as the PLATO goggles opened, cueing the participant to produce a MSE with the right hand by opening their fingers near the start position to reproduce their perceived object size. The goggles remained open for 2,000 ms. The participant confirmed their MSE by pressing a button on a 
keypad held in the left hand. The goggles closed at this point if the viewing duration had not already expired. The participant then pinched the fingers together at the starting position to null the grip aperture. After a 1,500 -ms delay, with random jitter sampled uniformly from $[-250,250] \mathrm{ms}$, the goggles again opened for 2,000 ms, upon which the participant grasped the target object and placed it at a marked location beyond the display, then returned the hand to the start position. If the viewing duration had not already expired by the time the thumb passed beyond the far edge of the display, the goggles closed. If the participant failed to make both responses on a given trial, the experimenter repeated the trial. The experimenter then set up the next display and pressed a button to trigger the next trial. The target's vertical position in the display was randomly jittered within the interval $[-1.5,1.5 \mathrm{~cm}]$ to encourage participants to look at the extent of the target object rather than the positions of the endpoints in relation to grid vertices in the display.

\section{Experiment phases}

There were four phases in the experiment, where the first three comprised the main experimental series (baseline, illusion-adaptation, and washout; see Fig. 2). The fourth phase (perceptually equalized phase) was designed to test the spatial separation hypothesis. During baseline, participants completed 20 trials (two locations by five sizes by two bins) on the neutral grid background. In the illusion-adaptation phase, the Ponzo display was introduced and participants completed 37 experimental trials (two locations by three sizes by six bins, plus one primer trial). In the washout phase, the neutral background was reintroduced, and participants completed 20 trials, just like the baseline phase. In the perceptually equalized phase, participants completed 16 trials, again with the Ponzo display (one location by two illusion contexts by two sizes by four bins).

\section{Trial order}

For the baseline and washout phases, two 10-trial bins were randomly constructed. For the illusion-adaptation phase, the trial order was determined by randomly ordering the six different preconstructed bins. During the illusion-adaptation phase, the first trial was a primer trial whose identity depended on the participant's group. This trial was needed so that the first trial of the first bin could be included in the previous-trial effect analysis. A participant's group also determined which six bins the participant observed during illusion-adaptation (out of a total of 12 used in the experiment). The trial sequence of each bin ensured that each participant experienced each of the four types of illusion transition at least once. The three physical object sizes appeared in a random order in each bin, with each size appearing once in each context. During the perceptually equalized phase, the trial sequence strictly alternated between the illusory-large and illusory-small displays, with the order of the sizes determined randomly within each of the four bins. The participant's group determined which context was presented first.

\section{Preregistered analysis}

\section{Preprocessing}

Trajectory data were linearly interpolated to fill missing frames and then smoothed using a second-order Butterworth filter with

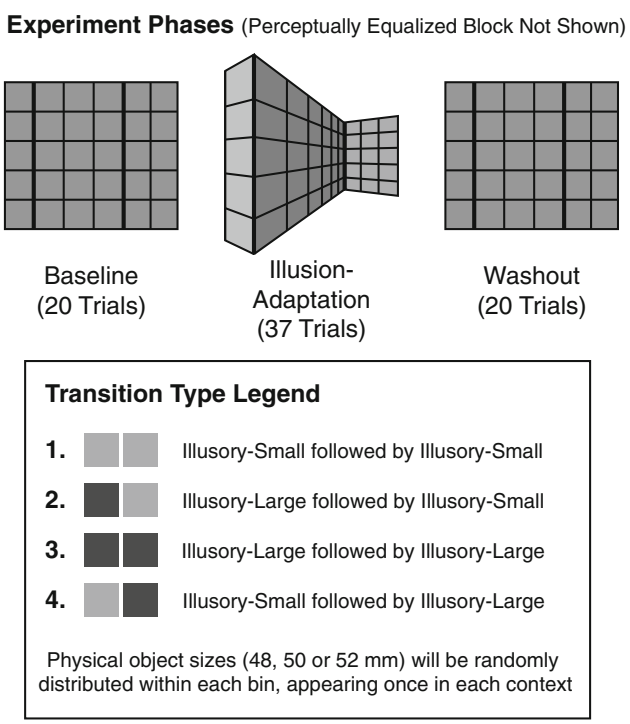

Fig. 2 Experiment phases and design of trial bins for the illusionadaptation phase. The main experimental series involved three phases (perceptually equalized phase not shown). During the illusionadaptation phase, each participant observed a trial sequence determined

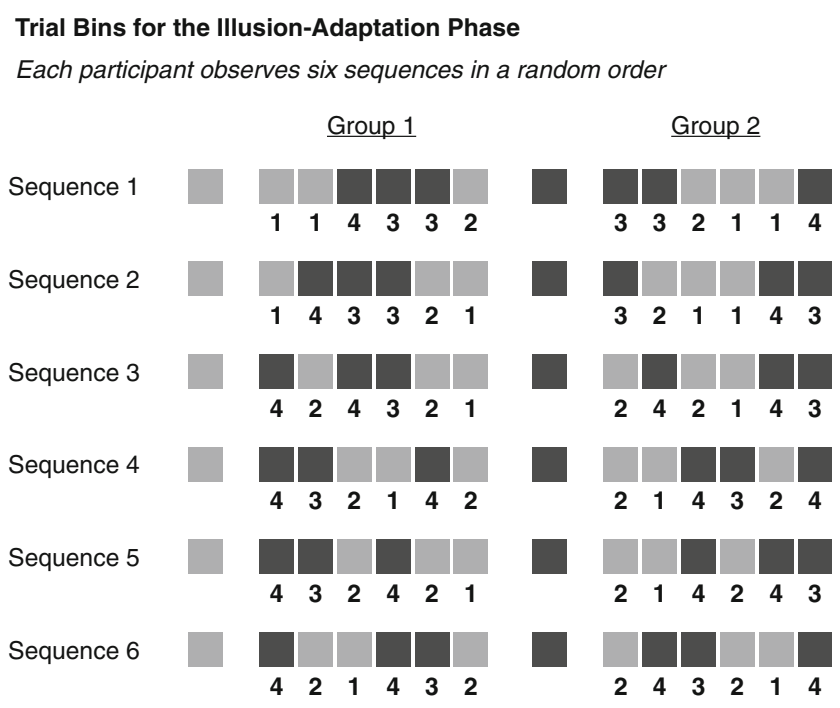

by randomly ordering six different trial bins (following one trial that primes the sequence, represented here by the offset leftmost square). These bins were constructed to allow balanced analysis of the previoustrial effect in each bin 
a cutoff frequency of $20 \mathrm{~Hz}$. The trajectories provided a continuous measure of the grip aperture from the start of the grasp response, which we planned to define as the frame on which the PLATO goggles opened, to object contact, which we planned to define by local minimization of hand velocity when the grip center was less than $3 \mathrm{~cm}$ from the target center. The MGA was extracted from the truncated trajectory.

\section{Data exclusion}

Individual MSE and MGA responses were excluded from the analysis and replaced with the participant's cell mean for that response and trial type (a) when they were more than 4 standard deviations above or below the participant's cell mean or (b) if failure of the automated preprocessing script followed by visual inspection of grasp profiles revealed that the trial was clearly anomalous. We planned to exclude participants from the analysis and replace them only in the event that the experiment was not completed (e.g., participant chooses to stop, equipment malfunction).

\section{Computing and testing illusion effects}

We planned to compute overall and binned illusion effects on MSEs and MGAs by taking the difference between the average responses to the illusory-large and the illusory-small targets within the corresponding trial windows and subtracting the mean difference in physical size across the two locations (this was a null difference in the main experimental series but was frequently nonnull in the perceptually equalized phase). When comparing MSEs to MGAs, we divided illusion effects by the average slopes of the MGA and MSE response functions in relevant temporal windows, determining slopes by fitting linear regressions over physical sizes. Standard errors of slope-corrected means were computed using the Taylorapproximation method (Franz, 2007; Kopiske et al., 2016). To analyze overall illusion effects during the illusionadaptation phase, we used a two-way (Physical Size $\times$ Response) ANOVA. We also tested the Pearson correlation between the mean illusion effects on MSE and MGA in the first two illusion-adaptation bins. To test the decrement in the illusion effect, we used a one-tailed paired-samples $t$ test to compare the uncorrected illusion effects on MGA in the first illusion-adaptation bin to that in the last illusion-adaptation bin. To test the previous-trial effect, we used a one-tailed paired-samples $t$ test to compare the MGAs of postillusorylarge grasps to the MGAs of postillusory-small grasps in the first illusion-adaptation bin. To test the hypothesis that consistent spatial separation of the opposing illusory contexts allows internal states to be maintained separately, we used a one-tailed paired-samples $t$ test to compare the illusion effect in the perceptually equalized phase to the illusion effect in the illusion-adaptation phase. Finally, to test whether there was a reduction in the MGA slope over the illusion-adaptation phase, we used a one-tailed paired-samples $t$ test to compare the slopes in the first bin to those in the last bin.

\section{Linear state-space model}

The visuomotor adaptation hypothesis can be formalized with a linear state-space model (Cheng \& Sabes, 2006; Thoroughman \& Shadmehr, 2000), which we applied to each participant's MGA sequences from the main experimental series. The model simulates grasp planning with a linear mapping from visual size $\left(\psi_{t}\right)$ to MGA:

$M G A_{t}=\alpha^{*} \psi_{t}+\beta+x_{t}$,

where $\alpha$ is a fixed slope parameter that defines how each individual's MGAs respond to target size changes and $\beta$ is a fixed intercept parameter. For each participant, these two parameters are estimated by fitting a linear regression over physical object sizes for all 93 trials. The additional offset parameter in Equation 1 is the current internal state $\left(x_{t}\right)$, which is the key variable controlled by the model. The model assumes two internal states, one controlling grasps to the left target and the other controlling grasps to the right target. The visual size of the target is defined as the sum of the physical size $\left(\varphi_{t}\right)$ and illusory distortion $\left(\omega^{S m}\right.$ or $\omega^{L g}$, fit for each individual):

$\psi_{t}=\varphi_{t}+\omega^{S m}$

or

$\psi_{t}=\varphi_{t}+\omega^{L g}$

When an illusory size estimate is used as the input to grasp control, sensorimotor feedback received during movement execution is assumed to allow for error detection. We operationalize the error signal as the difference between the produced MGA and each individual's "ideal MGA" for the current trial $(m)$ :

$e_{t}=M G A_{t}-m$,

where $m$ is estimated by inputting the physical object size to the participant's regression equation. Following error detection, a learning rule updates the internal states by partially decaying them and extracting from them some of the detected error for the current target ( $e_{t}^{S m}$ or $e_{t}^{L g}$, one of which is set to zero):

$\left[\begin{array}{c}x_{t+1}^{L g} \\ x_{t+1}^{S m}\end{array}\right]=A\left[\begin{array}{c}x_{t}^{L g} \\ x_{t}^{S m}\end{array}\right]-B\left[\begin{array}{c}e_{t}^{L g} \\ e_{t}^{S m}\end{array}\right]$ 
where $A=\left[\begin{array}{cc}a & 0 \\ 0 & a\end{array}\right]$ and $B=\left[\begin{array}{ll}b^{C} & b^{G} \\ b^{G} & b^{C}\end{array}\right]$. The first term, which is dependent on the current internal states, allows for some degree of state retention, with both states decaying at the same rate $a$. The second term, dependent on the observed error, governs how errors are extracted from the internal states. The diagonal terms, $b^{C}$, capture the degree of error correction specific to the target location, while the off-diagonal terms, $b^{G}$, capture the degree of generalization in error correction to the other target location. In other words, if $b^{G}$ is nonzero, then the state for the illusory-small object is also adjusted when an error is detected while grasping the illusory-large object, and vice versa.

This model was fit to minimize the root mean square error (RMSE) between the model simulation and participant data from the main experimental series using the COBYLA algorithm (Powell, 1998) of the nloptr package (Johnson, n.d.) in $\mathrm{R}$ ( $\mathrm{R}$ Core Team, 2014). The retention factor $(a)$ and correction parameters (constrained such that $b^{C} \geq b^{G}$ ) were bounded between -1 and 1 , and the distortions $\left(\omega^{S m}\right.$ and $\left.\omega^{L g}\right)$ were bounded between -5 and 5 . The final results of the model used initial guesses that, according to a coarse grid search, minimized the mean RMSE across all participants, but the initial parameter guesses were also randomized to ensure the reliability of the fit. To test for a significant illusion effect in the model fit, we used a one-tailed $t$ test to determine whether $\left(\omega^{L g}-\omega^{S m}\right)$ was positive. To test the location-specificity hypothesis, we used a one-tailed paired-samples $t$ test to determine whether $b^{C}$ exceeded $b^{G}$ in the main experimental series.

The model predictions displayed in Fig. 3 were based on simulations using estimates obtained from our pilot data ( $a=$ $0.974, b^{C}=0.654, b^{G}=0.341$ ), an estimated illusion effect of $2 \mathrm{~mm}\left(\omega^{S m}=-1.0, \omega^{L g}=1.0\right)$, and a baseline MGA response function with a slope of .82 and a 20 -mm intercept. The model predictions for the perceptually equalized phase used $b^{C}=b^{G}$ $=0.654-0.341=0.313$, since the absolute difference between these parameters primarily determines the adaptation rate, and we predicted the lack of spatial separation in this phase to cause full interference, equivalent to maintenance of a single state.

\section{Pilot data}

A pilot study with 45 participants provided preliminary support for our predictions. Here, we briefly present the results of this study, which was similar in design to the planned study. Participants viewed two 40 - by 8 - $\mathrm{mm}$ cylindrical dowels, attached with magnets to an upright display of the Ponzo illusion at positions $12 \mathrm{~cm}$ apart. On each trial, an audio clip cued the position of the target. Participants then performed manual size estimation followed by a grasping movement.
Subjects practiced the task during a training phase with square wooden targets on a blank white display. Natural vision and postural adjustments were allowed throughout the whole experiment. Instead of one marker on each digit, we used threeIRED clusters on small posts for data registration. We caution against a direct comparison of the illusion effect magnitudes in perception and grasping because the slopes of the MSE and MGA responses with respect to physical size are unknown due to the use of a single object size.

\section{Illusion effects on perception and action}

These data show overall illusion effects on both perception and grasping (see Fig. 4, center), with illusion effects on MSE that were correlated with the illusion effects on MGA across participants (Fig. 4, left), suggesting that the effect on grasping was driven by the same distorted size estimate as the perceptual effect. Figure 4 (right) depicts a binned analysis of the illusion effects on MSE and MGA across five four-trial bins - each illusion context was presented at least once within each bin. While illusion effects on perception and grasping are comparable in magnitude for the first two bins, the responses then diverge. Only at the end of the experiment did perception and grasping become dissociated.

The pilot study did not use trial sequences explicitly designed to measure the previous-trial effect, but we were able to do so by balancing two constraints: (1) minimize the size of the trial window, which necessarily begins on the second trial because the first had no predecessor, and (2) maximize the number of participants who saw all four transition types within this window. This manual optimization led us to use Trials 2 through 6 to assess the previous-trial effect. We found that MGAs following a grasp to the illusory-small object were larger than MGAs following a grasp to the illusory-large object (see Fig. 5). It is unclear why this effect $(2.7 \mathrm{~mm})$ was larger than our model predicts (see Fig. 2). Since it was even larger than the perceptual effect, we suspect that it is a result of noisy data (less than half of the participants could be used for this analysis).

Finally, we fit a simplified version of the model to the pilot data. Since we used only a single object size, each participant was assumed to have a MGA slope of 1.0 and the "deal MGA" was simply estimated as the mean MGA over all trials. The model estimated (1) a high degree of learning retention $(a=0.974,95 \% \mathrm{CI}=[0.963,0.984]),(2)$ significant error correction applied to both illusory distortions $\left(b^{C}=0.654\right.$, $95 \% \mathrm{CI}=[0.545,0.762])$, and (3) significant generalization of the error correction $\left(b^{G}=0.341,95 \% \mathrm{CI}=[0.189,0.493]\right)$. The model fit moderately captured the decrement in the illusion effect over time (Fig. 4, bottom right). Additionally, the estimated perceptual distortion was in line with that observed in the MSEs $\left(\omega^{L g}-\omega^{S m}=1.253,95 \% \mathrm{CI}=[0.228,2.278]\right)$, 
A

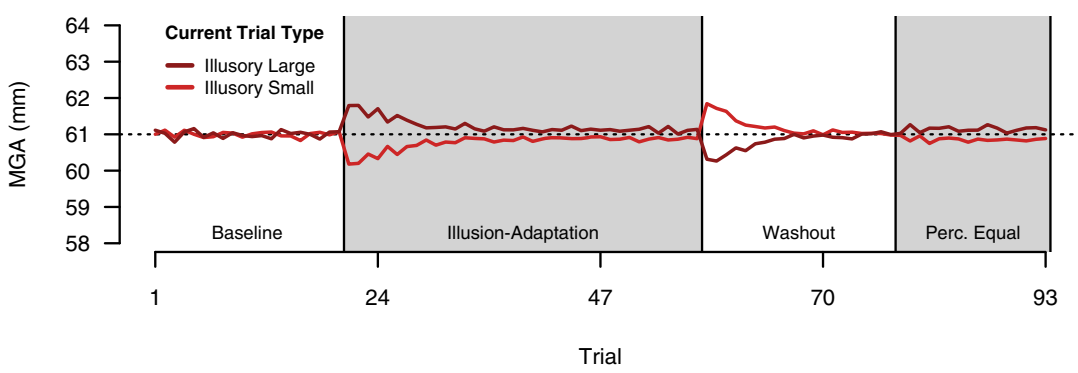

\section{B}

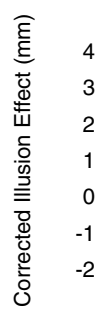

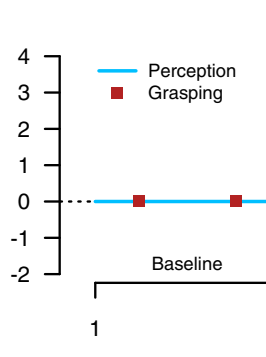

Model Prediction: Trial-by-trial MGAs

Model Prediction: Binned Illusion Effects

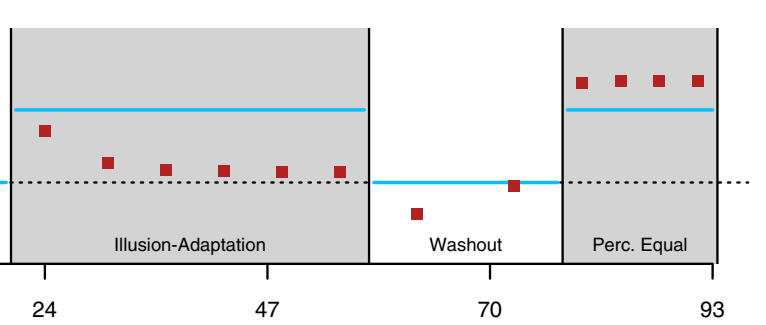

C

Model Prediction: Previous-Trial Effect Bin 1 of Illusion-Adaptation Phase

$$
\text { Trial }
$$

Fig. 3 Quantitative predictions of the adaptation model. a Average simulated MGAs for illusory-large (dark red) and illusory-small (red) targets, computed from 2,000 model simulations. Gray regions indicate when the Ponzo display is presented. There was no random error term in the simulated MGA response function-visible noise in these curves is due entirely to randomness in trial sequence generation and remains despite the large number of simulations. This figure was intended only as a demonstration of model behavior, not as an explicit prediction of the MGAs. We expected between-subjects variability and motor noise to obscure this pattern. b Predicted binned illusion effects. In the baseline phase, the model predicted no difference in MSEs or MGAs between the left and right neutral display locations. In the illusion-adaptation phase,

though the model was not fit to any MSE data (Fig. 4, top right).

\section{Implementation of preregistered protocol}

We recruited 84 participants to perform the experiment. During data collection, we tested four more participants than the preregistered sample size of 80 because we expected that a few participant exclusions might occur; however, no individual participants were excluded and we retained the entire sample. By the anomalous-trial exclusion criterion, 35 grasps were excluded and replaced, out of a total of 7,812 trials. All of these exclusions involved one or both finger markers not being visible to the Optotrak camera for significant portions of the trial. By the 4-SD outlier exclusion criterion, four additional grasps and eight MSEs were excluded and replaced.

In data preprocessing, we opted to use an enhanced method for defining movement onset and offset after observing poor performance with the preregistered method. For movement onset, we selected the first frame where the grip center had moved more than $5 \mathrm{~mm}$ after the PLATO goggles opened, the model assumed a stable positive effect on MSEs (lines) and predicted an exponential decrement in the effect on MGAs (squares). In the washout phase, the model predicted an anti-illusory aftereffect on MGAs. In the perceptually equalized phase, where trials alternated between illusorylarge and illusory-small in a single location, the model predicted an enhanced illusion effect on MGAs. The proportional enhancement was approximately equal to the model's error correction rate parameter. The parameters used for these simulations are reported in the Pilot Data. c Model also predicted a previous-trial effect in the first illusion-adaptation bin, where MGAs are larger when they follow a grasp to an illusory-small target than when they follow a grasp to an illusory-large target. (Color figure online)

which removed the interval of the reaction time. The enhanced method for movement offset detection involved constructing an objective function that combined multiple sources of kinematic information in order to robustly pinpoint the final frame of the movement (Schot, Brenner, \& Smeets, 2010). Specifically, our objective function jointly (1) required that the two digits be within broad bounding regions centered on the target endpoints, $(2 \mathrm{a})$ required that the grip aperture be greater than $46 \mathrm{~mm}$ (the minimum object size) then (2b) minimized the grip aperture, (3) minimized the absolute grip aperture velocity, and (4) minimized the $y$-coordinate (height) of the grip midpoint. The movement-offset frame was chosen according to the argmax of this function.

Two issues arose when computing illusion effects that led to deviations from the preregistered protocol. The first was in the computation of illusion effects on grasping in the illusion-adaptation phase, where we found that baseline-correction yielded results with reduced noise. The preregistered protocol involved computing combined illusion effects as differences across the left and right target locations, relying on display orientation counterbalancing to average out biomechanical biases 
Correlated Illusion Effects

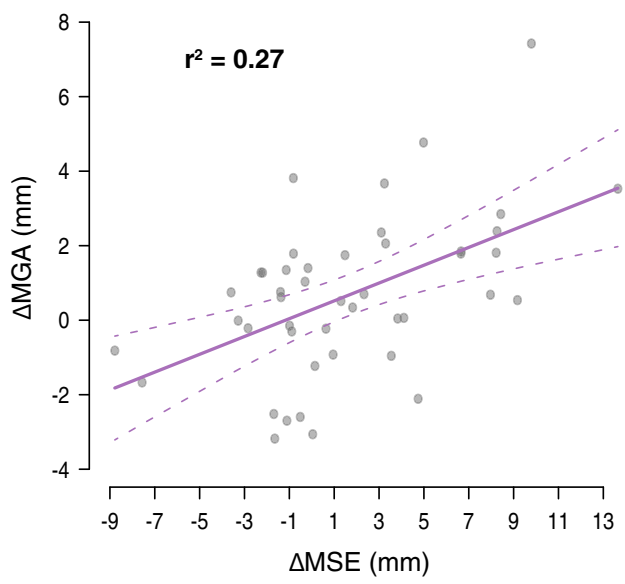

Fig. 4 Pilot study results $(N=45)$. The Ponzo illusion influenced both perception and grasping, and these effects were correlated, but only grasping seemed to become resistant to the illusion over 20 trials. Center: We found significant illusion effects on both manual size estimates ( $\triangle \mathrm{MSE})$ and maximum grip apertures ( $\triangle \mathrm{MGA})$. Left: Effects on perception and on grasping were positively correlated. Each $d o t$ is a participant. Participants who reliably reported the illusion via MSEs also tended to grasp according to the illusory sizes. Right: Evolution of $\Delta \mathrm{MSE}$ and $\triangle$ MGA over 20 trials, shown alongside average results from the

across the two locations. While the counterbalance was fairly successful in averaging out these biases, variance related to these biases remained in the data. Thus, it is preferable to baseline-correct the data, as it nullifies each participant's biomechanical bias by comparing illusionadaptation grasps to baseline grasps in the same location, effectively removing this source of variance. In this approach, the overall (combined) illusion effect is the difference between separately computed illusory-large and illusory-small effects. On the other hand, since MSEs were always produced near the start location, direct comparison across the two target locations presumably

Pilot Study Results

Previous-Trial Effect in Trials 2-6

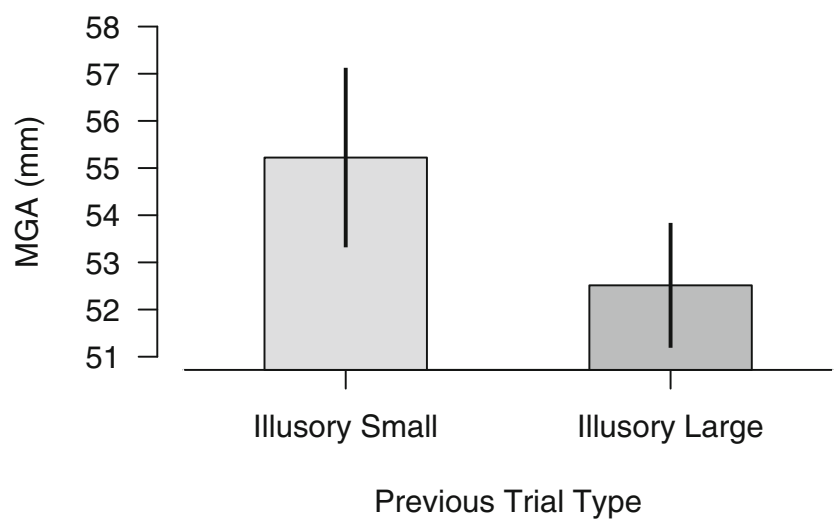

Fig. 5 Previous-trial effect on MGA in early trials of the pilot study

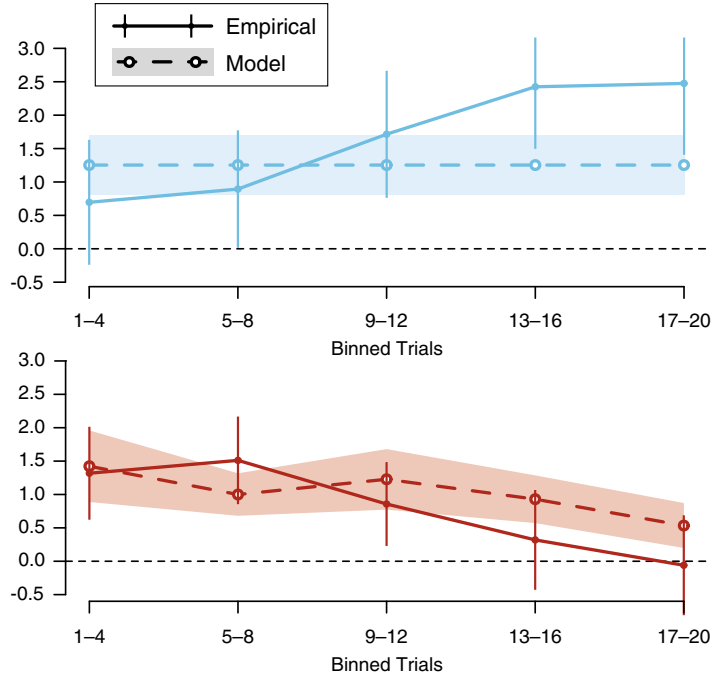

individual model fits. Top-right: The perceptual effect appears to have increased over the course of the experiment, perhaps because participants exaggerated or categorized their MSE responses with repeated trials. The model accurately estimated the mean perceptual effect from the MGA data. Bottom-right: Grasping was influenced by the illusion in the early trials but this effect gradually diminished over time $(n s)$. The model, which was fit to each participant's MGA time series by estimating size distortion and error correction rate parameters, roughly captures this pattern

introduces minimal variance related to individual biases. Baseline-correction was therefore not performed on MSE data; in the absence of position biases, the subtraction slightly increases variance.

Second, since the preregistered protocol incorrectly specified the procedure, it was necessary to deviate in the computation of illusion effects in the perceptually equalized phase. This phase involved a single target location for which we collected no baseline data, so there was neither the need nor the means to compute baselinecorrected effects. We computed slope-corrected illusion effects as differences between illusory-small and illusory-large responses, divided by the response slope. The response slope was estimated as the mean slope from separate linear regressions, one for each illusion context, over the two physical object sizes. Next, since the physical sizes of the targets in the two illusion contexts were often different, we computed the physical-size difference across the illusion contexts and subtracted this difference from the slope-corrected effects.

\section{Results}

All results reported in this section are based on preregistered analyses; the results of post hoc analyses are included solely in the Discussion. Tests are reported using traditional frequentist statistics as well as Bayes factors. Briefly, Bayes factors (BF) are ratios of the likelihood of the data given the null 
hypothesis (H0) to the likelihood of the data given the alternative hypothesis $(\mathrm{H} 1)$. In contrast to traditional frequentist tests, Bayes factors can be interpreted as evidence for $\mathrm{H} 1$ or H0, according to the Jeffreys (1961) guidelines: values less than 0.01 constitute strong evidence for $\mathrm{H} 0$; values between 0.01 and 0.33 constitute substantial evidence for $\mathrm{H} 0$; values between 0.33 and 3 are inconclusive; values between 3 and 10 constitute substantial evidence for $\mathrm{H} 1$; and values greater than 10 constitute strong evidence for H1. For detailed definitions of the $\mathrm{H} 0$ and $\mathrm{H} 1$ priors for each computed Bayes factor, see Table 1. All Bayes factors were computed using the Dienes (2008) calculator.

\section{Analysis of illusion effect dissociation}

The Ponzo illusion had a strong effect on perceptual responses (MSEs) throughout the illusion-adaptation phase, yet it did not have a detectable influence on grasping responses, even in the very first illusion-adaptation bin, $t(83)=1.40, p=0.082, \mathrm{BF}=$ 0.01 ; see Fig 6). We used a two-way (Physical Size $\times$ Response) ANOVA to compare the slope-corrected illusion effects on perception and grasping across all trials of the Illusion-Adaptation phase, applying the formula derived by Hesse, Franz, and Schenk (2016) to compute the individual slope-corrected illusion effects needed for ANOVA. A significant main effect of response confirmed that the illusion effect on MSEs was greater than on MGAs, $F(1,83)=28.90, p<$ $.001 ; \mathrm{BF}>10,000)$. The main effect of physical size was not significant, nor was the interaction of Response $\times$ Physical Size. Although illusion effects on MSE and MGA differed in magnitude, they were slightly correlated across individuals in the first two bins of illusion-adaptation $(r=0.24), t(82)=$ $2.25, p=0.027 ; \mathrm{BF}=3.75$.

\section{Analysis of effects predicted by adaptation model}

The main prediction of the adaptation model was that the illusion effect on MGA would decrease over the course of the illusion-adaptation phase. This prediction was not supported, as we did not detect a significant decrement in the MGA effect from the first bin to the last bin of illusion-adaptation, $t(83)=1.29, p=0.10, \mathrm{BF}=3.55$. A secondary prediction was that the illusion effect on MGA would be greater in the perceptually equalized phase than in the illusion-adaptation phase. We slope-corrected the MGA effects for this analysis because slopes were significantly lower in the perceptually equalized phase than in the illusion-adaptation phase (twotailed paired-samples $t$ test), $t(83)=2.62, p=.010$. This secondary prediction was also not supported, as the MGA effect was not found to be greater in the perceptually equalized phase, $t(83)=-0.80, \mathrm{p}=0.79, \mathrm{BF}=0.10$. Additionally, the corrected MSE effect remained significantly greater than the corrected MGA effect during the perceptually equalized phase, $t(83)=3.70, p<.001, \mathrm{BF}>1,000$. Although our two main predictions were not supported, we did find support for the third key prediction of the adaptation model: a significant previous-trial effect in the first bin of illusion-adaptation, $t(83)$ $=2.19, p=0.016, \mathrm{BF}=15.15$, which remained significant in a follow-up analysis of the entire illusion-adaptation phase, $t(83)=2.44, p=0.0084$ (see Fig. 7).

Finally, the two-state adaptation model was fit to the data from the first three phases, but none of the parameter estimates were meaningful, which is unsurprising given the null result for illusion effects on MGA (retention parameter $a=.88,95 \%$ $\mathrm{CI}=[0.83,0.91]$; error-correction parameter $b^{C}=-0.013$, $95 \% \mathrm{CI}=[-0.057,0.022]$; error-correction generalization parameter $b^{G}=-0.040,95 \% \mathrm{CI}=[-0.071,-0.013]$; estimated illusion effect $\omega^{L g}-\omega^{S m}=0.352,95 \%$ CI $=[-0.287,1.016]$; $b^{C}-b^{G}=0.027,95 \% \mathrm{CI}=[-0.01,0.063]$; confidence intervals computed using standard $\mathrm{BC}_{\mathrm{a}}$ bootstrap; Efron \& Tibshirani, 1993).

\section{Discussion}

In this preregistered study, we failed to replicate the decrement in a Ponzo illusion effect on closed-loop grasping that was found both in our pilot study and in an earlier study by Whitwell et al. (2016). The MGAs of grasping movements were never detectably biased by the illusion, even in the first bin of illusion-adaptation. We even failed to find a significant illusion effect in the first trial of illusion-adaptation (onetailed Welch's $t$ test), $t(81.72)=1.01, p=0.16$, achieved pow$\mathrm{er}=0.26$, so it was not the size of our trial bins that prevented us from observing adaptation. However, it is worth noting that the mean effects in that first trial went in the predicted directions $(0.48-\mathrm{mm}$ decrease for illusory-small, $0.44-\mathrm{mm}$ increase for illusory-large). Since MGAs were never detectably biased by the illusion, it is unsurprising that the parameter estimates from the model fit provided no evidence of error correction. There were simply no reliable differences in the magnitudes of the MGAs that our model could pick up on to drive trial-bytrial adjustments. Therefore, we must reject our initial hypothesis: visuomotor adaptation is apparently not needed to produce illusion-resistant MGAs during closed-loop grasping.

\section{What was the source of illusion-resistant MGAs?}

As noted in the Predictions section, finding illusion-resistant MGAs in the earliest trials of a closed-loop grasping task does not reveal much about the source of the illusion resistance. While the finding is certainly consistent with veridical vision-for-action, it is not inconsistent with the common representation model because there was a clear opportunity in this study for online control processes to fine-tune the grip aperture prior to the MGA. It is widely recognized that human 


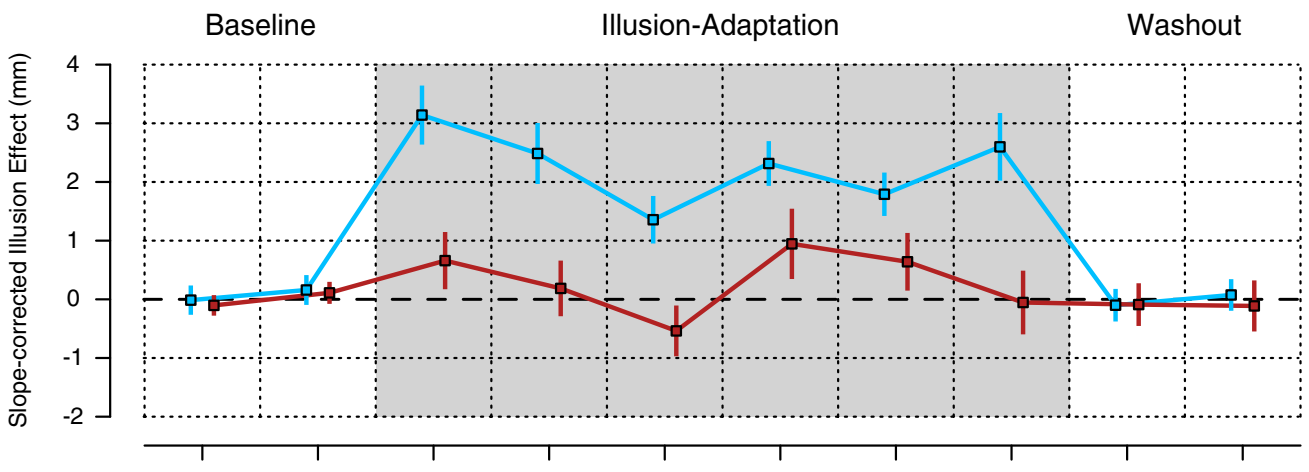

Perceptually Equalized
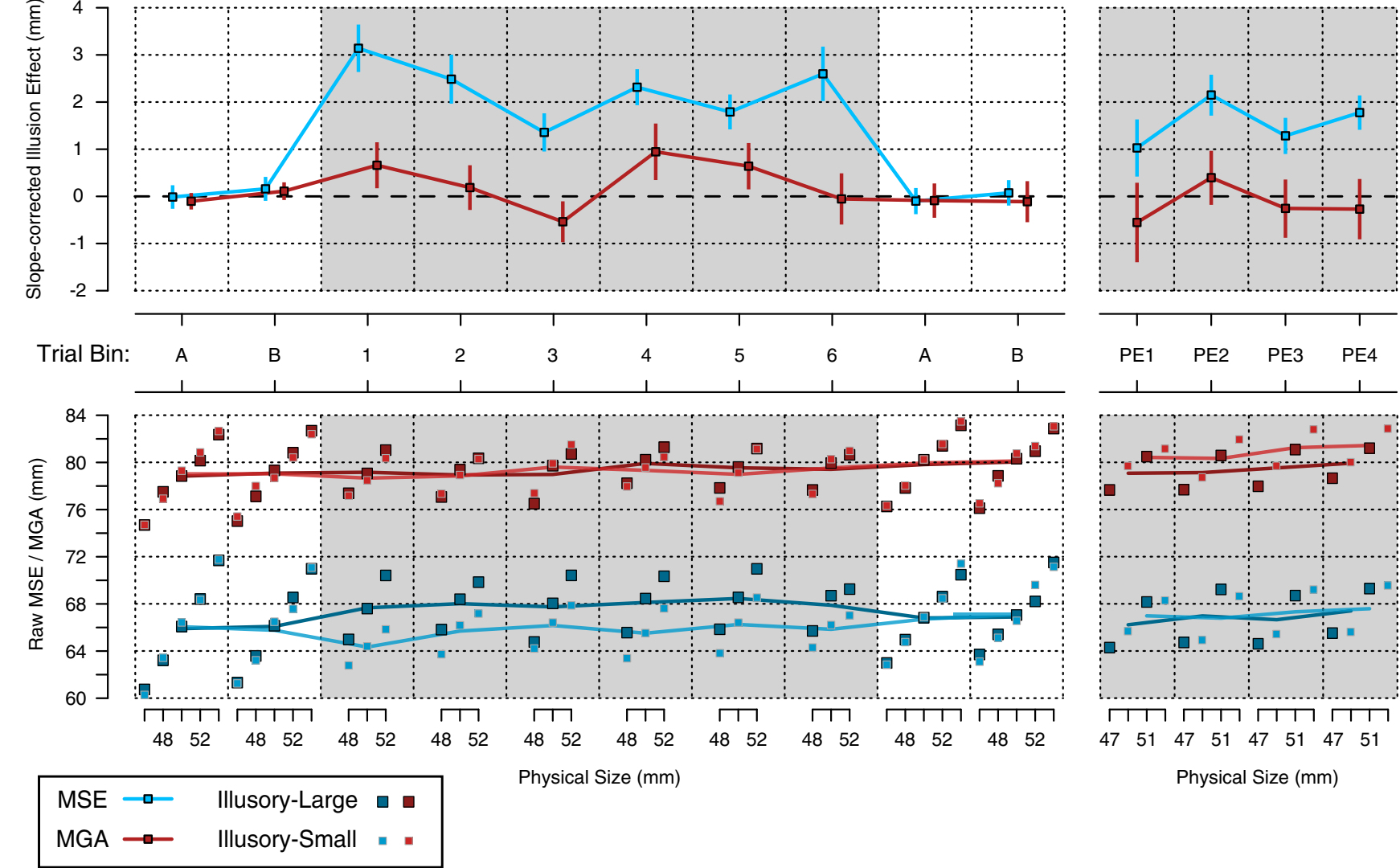

Fig. 6 Temporal analysis of illusion effects on MGA and MSE. (Top) Slope-corrected illusion effects by bin. (Bottom) Average MGA and MSE for each combination of illusion context and physical size. Solid lines trace the bin averages. Note. In the perceptually equalized phase (far right), targets in the illusory-small context were physically larger than targets in the illusory-large context by approximately $2 \mathrm{~mm}$. This was accounted for in the illusion effect calculation. (Color figure online)

loop tasks, whereas open-loop tasks yield effects on grasping that are comparable to effects on perception (Bruno \& Franz, 2009). Thus, we opted to conduct an exploratory post-hoc analysis aimed at evaluating whether the data are more consistent with online control or veridical vision-for-action. illusion effects on grasping are dramatically reduced in closed-

\section{Previous-Trial Effect on MGA}

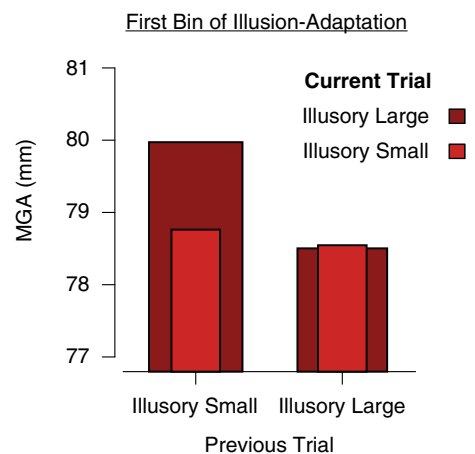

Fig. 7 Previous-trial effect on MGA. (Left) A significant effect was observed in Bin 1, as predicted by the adaptation model. (Right) The effect was also found when pooling data across the whole illusion-
All Six Bins of Illusion-Adaptation

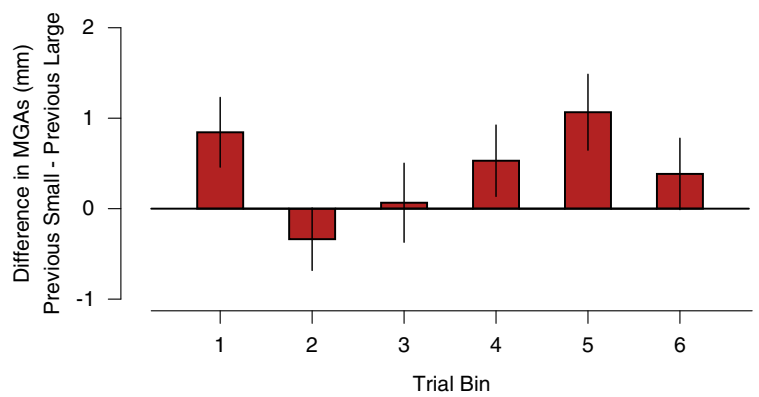

adaptation phase. A post hoc test also revealed a previous-trial effect on MSE (not shown; see Discussion). (Color figure online) 
Although this approach is similar to that of Glover and Dixon (2001a, 2002), we must distinguish the online control hypothesis investigated here from the planning-control model that they have defended. The critical difference is that the planning-control model maintains the notion of veridical vision-for-action by suggesting that online corrections also occur in open-loop conditions, a claim that has been challenged by Franz, Scharnowski, and Gegenfurtner (2005).

We first explored whether summary statistics on basic spatiotemporal characteristics of the grasping movements might rule out the possibility of online control. We found that the average distance traveled by the grip midpoint from MGA to movement offset was $18.3 \mathrm{~mm}$, the average movement duration was $715 \mathrm{~ms}$, the average velocity was $0.35 \mathrm{~m} / \mathrm{s}$, and the average peak velocity was $0.70 \mathrm{~m} / \mathrm{s}$. None of these results suggest ballistic, uncontrolled movement. Considering the extremely close proximity of the hand and the target at MGA, it would be surprising if the control system did not make use of this opportunity to fine-tune the relative positions of the fingers and the contact points.

\section{Grip aperture trajectory analysis}

Next, we performed an exploratory analysis of grip aperture trajectories. Trajectory analyses must be conducted by aligning movement trajectories along a temporal (e.g., elapsed time) or spatial (e.g., distance traveled) ordinate dimension, and the chosen dimension may be either landmark oriented (e.g., setting movement onset, peak velocity, MGA, or movement offset as the origin) or normalized between two landmarks (e.g., aligning trajectories at movement onset and movement offset so they span arbitrary units from zero to one). It is important to be cautious in this endeavor, as previous studies have shown that the choice of ordinate variable can dramatically influence the results of trajectory analysis (Smeets, Glover, \& Brenner, 2003; Whitwell \& Goodale, 2013). This is because the ordinate variable determines how the discrete points along one trajectory match up with the discrete points along a comparison trajectory.

The dramatic example analyzed by Whitwell and Goodale (2013, see their Fig. 1) is helpful in understanding why certain choices can produce artifacts. Imagine that the MGAs of two grasping movements are identical and occur at the same absolute time, but that the closure phase is slightly longer for one movement because it is directed at a smaller target, requiring additional time for the fingers to close. In this case, time normalization will make it look like the grip aperture trajectories proceeded differently from movement onset. Fortunately, this artifact would easily be revealed by analyzing the data with other ordinate variables, such as absolute elapsed time or, assuming the MGAs occur at roughly the same spatial proximity to the target, distance to contact.
We emphasize that our trajectory analysis was robust across multiple choices of ordinate variable, including distance to contact, absolute elapsed time, absolute elapsed time relative to MGA, normalized elapsed time, distance traveled relative to MGA, and normalized distance traveled. This consistency makes sense given the fact that there were no appreciable differences across the two illusion contexts in the overall movement durations or distances, nor in the time or location at which the MGA occurred, which are the underlying factors that are known to produce spurious findings (Smeets et al., 2003; Whitwell \& Goodale, 2013).

We chose to align trajectories to the ordinate variable distance to contact, which was computed by tracing backwards from movement offset along the traveled path of the grip midpoint. We interpolated the grip aperture data of each individual trial at $1-\mathrm{mm}$ intervals spanning the final $220 \mathrm{~mm}$ of the movement. This was the maximum amount of the trajectory that we could analyze without dropping participants from the analysis; average movement length was $244 \mathrm{~mm}$. As was done with the MGA data, separate illusion effect profiles were computed for the illusory-large and illusory-small contexts via baselinecorrection and combined illusion effect profiles were computed as the difference between the separate illusion effect profiles. In the absence of a clear hypothesis regarding temporal changes in online control, we pooled together all six bins of illusion-adaptation for this analysis.

The top panels of Fig. 8 show the mean grip aperture profiles in baseline and illusion-adaptation for the two illusion contexts. In each panel, the two empirical profiles are displayed alongside an estimated planned profile. The estimated profiles were computed as follows: first, we derived a function that allowed us to estimate the trajectory of a grasp toward an object of any size in the tested range (46-54 $\mathrm{mm}$ ) by interpolating over baseline trajectories, then we used the slopecorrected effect on MSE to estimate the planned trajectory for two "imagined" objects that were increased or decreased in size by half of the perceptual effect. This generated a quantitative prediction for the online control hypothesis: the grip aperture profiles in the two illusory contexts would be expected to initially follow the estimated profiles, as if they were aiming at objects with physically increased or decreased sizes, but to diverge toward the baseline profiles before the MGA.

The middle panel of Fig. 8 shows two curves representing the separate illusory-large and illusory-small effect profiles, while the bottom panel of Fig. 8 shows the combined illusion effect profile. The estimated planned profiles were used to generate predictions for the separate illusion effect and the combined illusion effect, also displayed in the lower panels. As the hand traveled from the start position to a location about $150 \mathrm{~mm}$ from contact, the combined illusion effect gradually increased, closely following the predicted effect profile. Thereafter, the effect diminished, just as one would expect under the online 

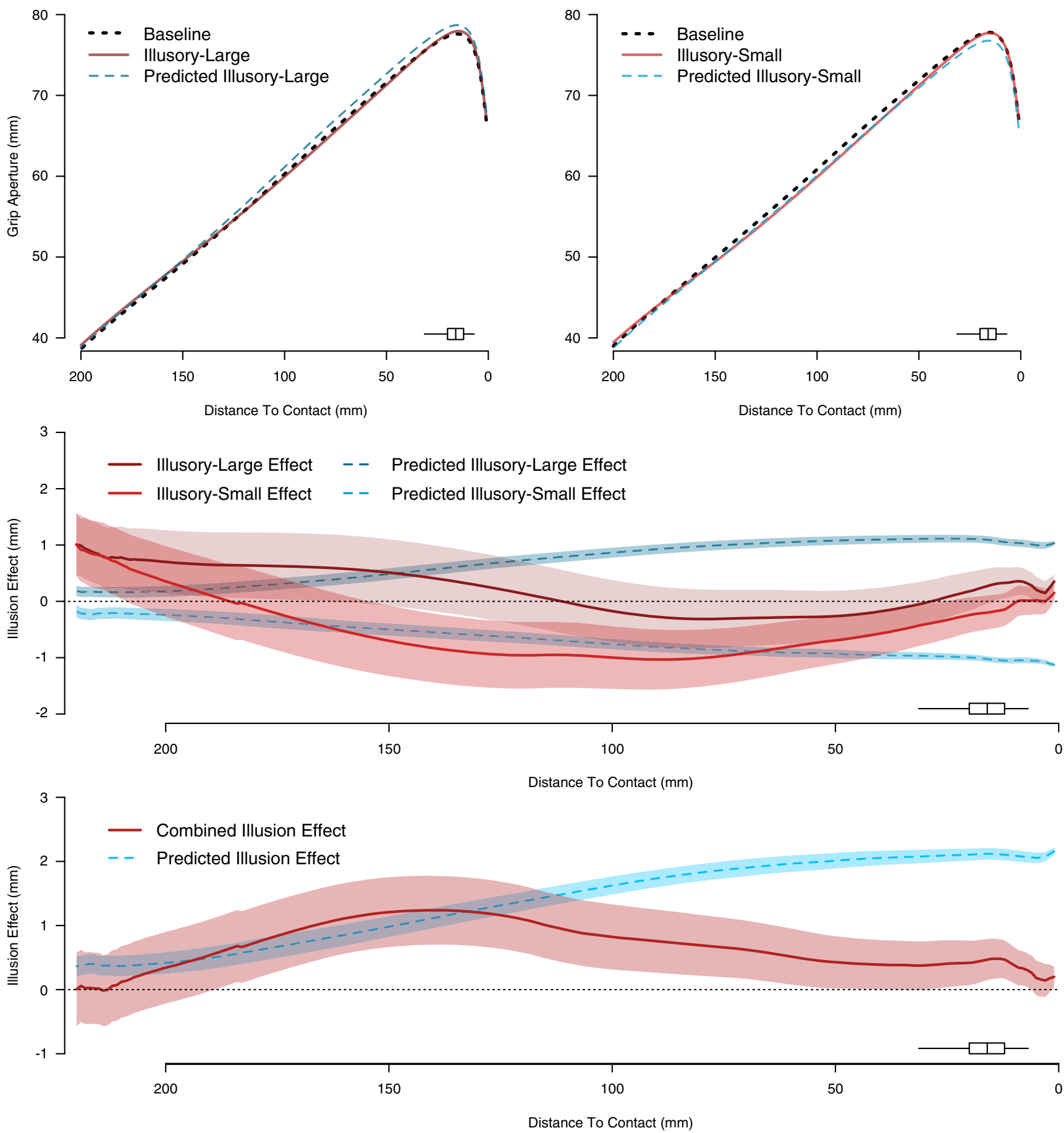

Fig. 8 Post hoc analysis of grip aperture trajectories in illusionadaptation phase. (Top) Grip aperture trajectories for the illusory-large (left) and illusory-small (right) contexts. Dotted black lines are average baseline grasp trajectories, specific to the location that the corresponding illusion context was presented. We used baseline grasp data to estimate what the trajectory should look like for two "imagined" targets differing in physical size by the magnitude of the MSE effect (blue dashed line). If grasping were completely deceived by the illusion, the grip aperture trajectory would be expected to match this estimate until just before contact.

control hypothesis. Overall, this exploratory analysis was more consistent with the online control account of
Red lines are average grip aperture trajectories over the entire illusionadaptation phase. Boxplots depict the spatial distribution of MGAs. (Middle) Separate illusion effect trajectories for illusory-large and illusory-small contexts, computed as the difference between the red and black lines depicted above. (Bottom) Combined illusion effect trajectory, computed as the difference between the separate illusion effect trajectories. This data evolves in line with the prediction until the hand reaches a point roughly $14 \mathrm{~cm}$ from contact, after which the effect is attenuated. (Color figure online)

illusion-resistant MGAs, as opposed to veridical visionfor-action. 


\section{Interpreting the previous-trial effect}

Despite finding illusion-resistant MGAs in the first illusionadaptation bin, which shows that illusion resistance is possible without visuomotor adaptation, one prediction of the adaptation model was borne out in the data: the illusory context presented on a given trial influenced the MGA on the next trial, in a direction opposite to the preceding illusory distortion (see Fig. 8). How could we have obtained this previous-trial effect, which was predicted as a consequence of trial-by-trial error correction, without also finding that the illusion caused significant errors at the MGA?

One possibility is that the previous-trial effect on MGA had nothing to do with the movement made on the previous trial. Rather, it may be the result of a perceptual or cognitive contrast between illusion contexts. A perceptual contrast would imply that the perceived size distortion is amplified when the illusion context is switched between trials and that this enhanced perceptual distortion did in fact affect MGAs. On the other hand, a cognitive contrast would imply that higher-level knowledge of the Ponzo illusion effect leads participants to slightly exaggerate their responses when the illusion context is switched. By this account, the previous-trial effect does not conflict with the perception-action dissociation of the TVSH. An issue with both accounts is that they predict a current-trial effect, which we did not find in our primary analysis. However, they also predict a strong previous-trial effect on perceptual responses, which was notably confirmed in a post hoc analysis of MSEs (one-tailed $t$ test), $t(83)=4.48, p<.001$.

A second possibility is that the previous-trial effect reflects the activity of a trial-by-trial motor learning mechanism that causes the grasp control system to counteract illusion-induced errors made on the previous trial. Our exploratory trajectory analysis showed that systematic movement errors did occur in early portions of the trajectory, corroborating the main assumption of this account. However, if the underlying motor learning process were a genuine error-correction process that affected grasp planning (as in our adaptation model), then the early-trajectory errors would be expected to decrease across the illusion-adaptation phase. In contrast, the early-trajectory illusion effect (computed for the whole illusion-adaptation phase) closely matched the effect predicted from the MSE data, indicating that no decrement occurred. To account for the lingering early-trajectory effect, the intertrial influence of the previous movement would have to have been limited to later movement stages. One way this could have occurred is through use-dependent learning, an important counterpart to error-based learning (Diedrichsen, White, Newman, \& Lally, 2010; Verstynen \& Sabes, 2011). By this process, the online correction performed on one trial could have encouraged a similar correction to be made on the next trial.

Both of the above interpretations make the additional prediction that larger illusion effects will occur on switched- context trials than on same-context trials (see the adaptation model prediction in Fig. 3c). In a post hoc analysis of MGAs, we found that switched-context trials did not produce significantly larger illusion effects than same-context trials (onetailed $t$ test), $t(83)=1.13$ ). However, when comparing MSE effects in switch versus same trials, we obtained a significant difference of $1.12 \mathrm{~mm}$ (one-tailed $t$ test), $t(83)=2.02, p=.023$. Taken together, the results slightly favor the perceptual or cognitive bias explanations because the effects on MSE were found to be quite robust, whereas the effects on MGA, which are central to the use-dependent plasticity explanation, were smaller and less reliable. Additionally, the idea that the usedependent plasticity explanation can also explain the previous-trial effect on MSEs, while plausible, is currently highly speculative.

\section{Why did we fail to replicate the decrement in the effect on grasping?}

The results of this study failed to replicate those of Whitwell et al. (2016) and our pilot study, both of which were closedloop tasks that elicited early-trial illusion effects on MGA. For reference, a visual summary of temporal illusion effect analyses reported in seven different experiments is displayed in Fig. 9, including three open-loop grasping experiments that used the Müller-Lyer and parallel lines illusions (Franz et al., 2001; Kopiske et al., 2017). Two trends are visible: (1) Ponzo MSE effects are fairly consistent, around $2 \mathrm{~mm} \approx 4 \%$ of target size; and (2) open-loop studies (which used different illusions) tend to elicit larger initial effects on MGA. In this final section, we focus on possible reasons for the contradiction between our result and the two comparable Ponzo illusion studies, drawing connections to related literature as needed.

\section{Carryover of control strategy from preceding task}

In the Whitwell et al. (2016) study and our pilot study, participants did not grasp the target objects during a baseline phase before the illusion was introduced, as they did in the present study. Thus, the extended baseline phase of the present study could have contributed to the observed illusion resistance in the first illusion-adaptation bin, as it provided an opportunity to optimize a feedback-based online control strategy (Elliott \& Lyons, 1998). On this account, if vision of the hand were not available during the baseline phase, then there would not have been suppression of the illusion effect. This prediction is borne out in the results of Kopiske, Cesanek, Campagnoli, and Domini (2017): Despite allowing extensive practice with open-loop grasping in a baseline phase, large illusion effects on MGA emerged when the Müller-Lyer illusion was presented (as shown in Fig. 9). This conflicts with the alternative hypothesis that baseline practice, as a means of making grasping movements more automatic and less controlled, engages 


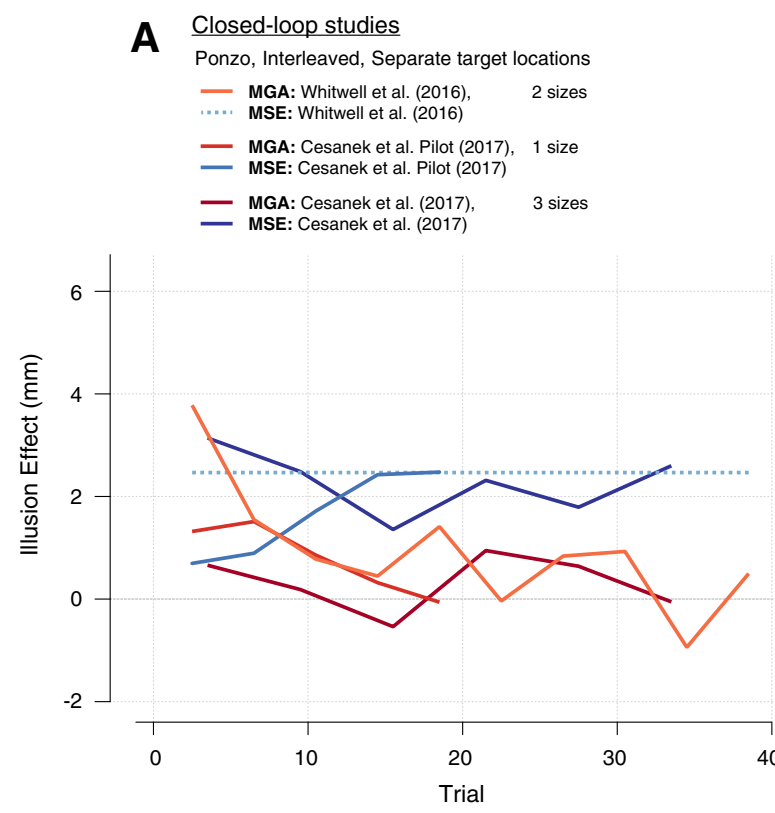

Fig. 9 Visual summary: Reported temporal analyses of illusion effects on MGA and MSE. a Closed-loop experiments used the Ponzo illusion and measured both MGA (reds) and MSE (blues). Whitwell et al. (2016) measured the MSE effect before and after the grasping block, here we show the mean (light-blue dashed line). MGA and MSE slopes were considerably less than 1 in Whitwell et al. (2016), so we slopecorrected the reported effects to improve comparability. Illusion effects

accurate dorsal stream visual processing (Goodale et al., 2008). Overall, these findings are consistent with the idea that sensorimotor networks naturally hone in on control strategies that make efficient use of available feedback.

Additionally, Whitwell et al. (2016) had participants perform a block of MSEs (without haptic feedback) before the grasping block. In that block, participants may have become accustomed to producing larger grips when responding to one side of the Ponzo display and smaller grip sizes for the other. Following the sudden transition into the grasping block, it would have been difficult to suppress an automatic tendency to make more dramatic movements when attending to the illusory-large region of the display. This is another possible source of the strong effect on MGA observed in the first grasping bin of that study, consistent with use-dependent motor learning (Diedrichsen et al. 2010; Verstynen \& Sabes, 2011).

\section{Effect size overestimation does not explain the non-replication}

A more mundane possibility for the nonreplication of the adaptation effect is that we overestimated the effect size in our power analysis. Indeed, our sample size was chosen based on the medium effect size estimated from Whitwell et al. (2016) rather than the small effect size estimated from our pilot study. However, an important point here is that the decrement effect size in adaptation is largely dictated by the effect size of the
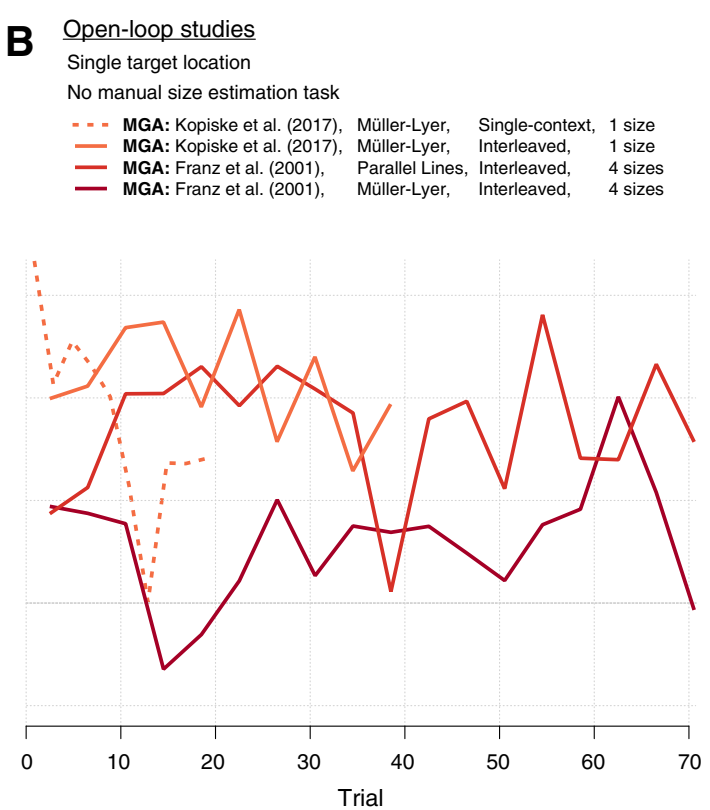

were already slope-corrected in the present study. Our pilot study used only one target size so response slopes are unknown. b Open-loop experiments used the Müller-Lyer or parallel lines illusion and measured only MGA, not MSE. Illusion effects were already slope-corrected in Kopiske et al. (2017). The MGA slope was nearly 1 in both Franz et al. (2001) experiments. Note the difference in the ordinate scale between Panels A and B. (Color figure online)

initial perturbation. A post hoc power analysis indicated that our achieved power for detecting the MGA effect in the first illusion-adaptation bin was 0.40 and in the first trial it was 0.26 . These results show that we may not have collected a large enough sample to detect a very small MGA effect in the first bin. At the same time, we observed a significantly smaller illusion effect on MGAs than on MSEs in the first illusion-adaptation bin, so it is clear that the initial illusion effect was reduced without the aid of the hypothesized visuomotor adaptation mechanism. Therefore, an overestimated effect size does not explain the key factor in the nonreplication: the source of the reduced illusion effect on MGAs.

\section{Is there still a potential role for adaptation?}

Although visuomotor adaptation was not responsible for illusion-resistant grasping in the present study, adaptation could still play a role in reducing illusion effects when grasping is strongly biased. The results of Kopiske et al. (2017) confirm this: An initial 6.6-mm effect of the Müller-Lyer illusion on open-loop MGAs decreased by more than $50 \%$ over 20 trials, and a negative aftereffect was observed when the illusory fins were removed from the target bars. Why, then, have a considerable number of studies been able to find strong effects on MGAs, with no apparent suppression from adaptation (e.g., , 2003; Franz et al., 2003; Franz et al., 2001; Franz 
et al., 2000; Franz et al., 2009; Kopiske et al., 2016 Pavani et al., 1999; Vishton et al., 1999)? For example, Franz et al. (2001) showed that the effect of the Müller-Lyer illusion on open-loop MGAs was greater than its effect on a perceptual judgment task, and showed no decrement over 72 trials. Kopiske et al. (2017) demonstrated that this is largely because of interference in adaptation, as discussed in the introduction of this study. When opposing illusion contexts were interleaved in a single block, they were unable to detect any decrement in the illusion effect on open-loop MGAs over 40 trials (see their interleaved and single-context conditions in Fig. 9). This suggests that trial-by-trial error corrections do occur in single-location open-loop studies, but that conflicting signals prevent the system from settling on an effective configuration.

A separate but related line of investigation has focused on decrements in illusion effects on traditional perceptual judgments, suggesting that there may be a perceptual component to illusion adaptation when there is continuous or repeated active visual exposure to the display (Coren \& Hoenig, 1972; Judd, 1902; Lewis, 1908; Festinger, White, \& Allyn, 1968). More recently, Predebon (2006) found that a continuous series of brief exposures to the illusion, as in most illusion and grasping studies, produced a notable decrement when the intertrial interval was shorter than 20 seconds. Our study involved less-than-10-second intertrial intervals of visual occlusion, thus providing suitable conditions for a perceptual decrement. In a post hoc analysis, we computed linear regression slopes across the six illusion-adaptation bins on individual slope-corrected MSE effects (again applying the formula of Hesse et al., 2016, pp. 94). The average change in the slopecorrected MSE effect per bin was $-0.11 \mathrm{~mm}$, but this slope was not significant, $t(83)=1.06$, so we cannot conclude that the perceived size distortions were attenuated. Moreover, a perceptual decrement does not seem to have occurred in the results of Whitwell et al. (2016), as MSE effects were relatively stable within-blocks and across the grasping block in their ABA design, nor can this explain the results of the pilot study, where an interaction was found in the temporal development of illusion effects on MGA and MSE. Overall, the apparent stability of the MSE effects in multiple studies (see Fig 9.) is somewhat surprising given the vast literature demonstrating perceptual illusion decrements under similar repeatedjudgment conditions.

\section{Conclusion}

This preregistered study was designed to test the predictions of a computational model of visuomotor adaptation that could potentially account for reduced illusion effects on grasping in studies involving repeated movements. Since we did not observe illusion effects on MGAs, even in the first trial of our illusion-adaptation phase, it is unsurprising that strong signatures of visuomotor adaptation were not observed in the time course of the MGAs nor in the model parameter estimates. Therefore, we conclude that visuomotor adaptation is not needed to explain the illusion resistance of closed-loop MGAs. This result is consistent with either (a) a veridical vision-for-action system or (b) a visuomotor system that makes automatic online corrections based on visual feedback to eliminate illusion effects prior to the MGA. An exploratory post hoc analysis of full movement trajectories favored the predictions of this online control explanation, suggesting that the Ponzo illusion did affect grasp planning.

\section{References}

Aglioti, S., DeSouza, J. F. X., \& Goodale, M. A. (1995). Size-contrast illusions deceive the eye but not the hand. Current Biology, 5(6), 679-685.

Bartelt, R., \& Darling, W. G. (2002). Opposite effects on perception and action induced by the Ponzo illusion. Experimental Brain Research, 146(4), 433-440.

Bingham, G., Coats, R., \& Mon-Williams, M. (2007). Natural prehension in trials without haptic feedback but only when calibration is allowed. Neuropsychologia, 45, 288-294.

Brenner, E., \& Smeets, J. B. J. (1996). Size illusion influences how we lift but not how we grasp an object. Experimental Brain Research, 11, 473-476.

Bruno, N., \& Franz, V. H. (2009). When is grasping affected by the Müller-Lyer illusion?: A quantitative review. Neuropsychologia, 47, 1421-1433.

Cheng, S., \& Sabes, P. N. (2006). Modeling sensorimotor learning with linear dynamical systems. Neural Computation, 18, 760-793.

Coren, S., \& Hoenig, P. (1972). Eye movements and decrement in the Oppel-Kundt illusion. Perception \& Psychophysics, 12(2B), 224 225.

Diedrichsen, J., White, O., Newman, D., \& Lally, N. (2010). Usedependent and error-based learning of motor behaviors. Journal of Neuroscience, 30(15), 5159-5166.

Dienes, Z. (2008). Understanding psychology as a science. An introduction to scientific and statistical inference. New York, NY: Palgrave Macmillan.

Dienes, Z. (2011). Bayesian versus orthodox statistics: Which side are you on? Perspectives on Psychological Science, 6(3), 274-290.

Dyde, R. T. \& Milner, A. D. (2002). Two illusions of perceived orientation: One fools all of the people some of the time; the other fools all of the people all of the time. Experimental Brain Research, 144(4), $518-527$.

Efron, B., \& Tibshirani, R.J. (1993). An introduction to the bootstrap. New York, NY: Chapman \& Hall.

Elliott, D., \& Lyons, J. (1998). Optimizing the use of vision during motor skill acquisition. in J. P. Piek (Ed.), Motor behavior and human skill: A multidisciplinary approach (pp. 57-72). Champaign, IL: Human Kinetics.

Festinger, L., White, C. W., \& Allyn, M. R. (1968). Eye movements and decrement in the Müller-Lyer illusion. Perception \& Psychophysics, 3, 376-382.

Flanagan, J. R., \& Beltzner, M. A. (2000). Independence of perceptual and sensorimotor predictions in the size-weight illusion. Nature Neuroscience, 3(7), 737-741. 
Flash, T., \& Hogan, N. (1985). The coordination of arm movements: An experimentally confirmed mathematical model. The Journal of Neuroscience, 5(7), 1688-1703.

Franz, V. H. (2003). Manual size estimation: a neuropsychological measure of perception? Experimental Brain Research, 151(4), 471-477.

Franz, V. H. (2007). Ratios: A short guide to confidence limits and proper use. Retrieved from http://arxiv.org/pdf/0710.2024v1.pdf

Franz, V. H., Bülthoff, H. H., \& Fahle, M. (2003). Grasp effects of the Ebbinghaus illusion: Obstacle-avoidance is not the explanation. Experimental Brain Research, 149, 470-477.

Franz, V. H., Fahle, M., Bülthoff, H. H., \& Gegenfurtner, K. R. (2001). Effects of visual illusions on grasping. Journal of Experimental Psychology: Human Perception \& Performance, 27(3), 1124-1144.

Franz, V. H. \& Gegenfurtner, K. R. (2008). Grasping visual illusions: Consistent data and no dissociation. Cognitive Neuropsychology, 25(7/8), 920-950.

Franz, V. H., Gegenfurtner, K. R., Bülthoff, H. H., \& Fahle, M. (2000). Grasping visual illusions: No evidence for a dissociation between perception and action. Psychological Science, 11(1), 20-25.

Franz, V. H., Hesse, C., \& Kollath, S. (2009). Visual illusions, delayed grasping, and memory: No shift from dorsal to ventral control. Neuropsychologia, 47(6), 1518-1531.

Franz, V. H., Scharnowski, F., \& Gegenfurtner, K. R. (2005). Illusion effects on grasping are temporally constant, not dynamic. Journal of Experimental Psychology: Human Perception and Performance, 31(6), 1359-1378.

Ganel, T., Tanzer, M., \& Goodale, M. A. (2008). A double dissociation between action and perception in the context of visual illusions: Opposite effects of real and illusory size. Psychological Science, 19(3), 221-225.

Ghahramani, Z., Wolpert, D. M., \& Jordan, M. I. (1996). Generalization to local remappings of the visuomotor coordinate transformation. The Journal of Neuroscience, 16(21), 7085-7096.

Glover, S. \& Dixon, P. (2001a). Dynamic Illusion effects in a reaching task: Evidence for separate visual representations in the planning and control of reaching. Journal of Experimental Psychology: Human Perception and Performance, 27, 560-572.

Glover, S., \& Dixon, P. (2001b). Motor adaptation to an optical illusion. Experimental Brain Research, 137(2), 254-258.

Glover, S., \& Dixon, P. (2002). Dynamic effects of the Ebbinghaus illusion in grasping: Support for a planning/control model of action. Perception \& Psychophysics, 64(2), 266-278.

Gonzalez, C. L. R., Ganel, T., Whitwell, R. L., Morrissey, B., \& Goodale, M. A. (2008). Practice makes perfect, but only with the right hand: Sensitivity to perceptual illusions with awkward grasps decreases with practice in the right but not the left hand. Neuropsychologia, 46, 624-631.

Goodale, M. A., Gonzalez, C.L.R, \& Kroliczak, G. (2008). Action rules: Why the visual control of reaching and grasping is not always influenced by perceptual illusions. Perception, 37, 355-366.

Goodale, M. A. \& Milner, A. D. (1992). Separate visual pathways for perception and action. Trends in Neurosciences, 15(1), 20-25.

Grandy, M. S. \& Westwood, D. A. (2006). Opposite perceptual and sensorimotor responses to a size-weight illusion. Journal of Neurophysiology, 95(6), 3887-3892.

Haffenden, A. M., \& Goodale, M. A. (1998). The effect of pictorial illusion on prehension and perception. Journal of Cognitive Neuroscience, 10(1), 122-136.

Haffenden, A. M., \& Goodale, M. A. (2000). Independent effects of pictorial displays on perception and action. Vision Research, 40, $1597-1607$.

Haffenden, A. M., Schiff, K. C., \& Goodale, M. A. (2001). The dissociation between perception and action in the Ebbinghaus illusion: Nonillusory effects of pictorial cues on grasp. Current Biology, 11(3), 177-181.
Hesse, C., Franz, V. H., \& Schenk, T. (2016). Pointing and anti-pointing in Müller-Lyer figures: Why illusion effects need to be scaled. Journal of Experimental Psychology: Human Perception and Performance, 42(1), 90-102.

Jackson, S. R., \& Shaw, A. (2000). The Ponzo illusion affects grip-force but not grip-aperture scaling during prehension movements. Journal of Experimental Psychology: Human Perception and Performance, 26(1), 418-423.

Jeannerod, M. (1984). The timing of natural prehension movement. Journal of Motor Behavior, 16(3), 235-254.

Jeffreys, H. (1961). Theory of probability. In N. F. Mott, E. C. Bullard, \& D. H. Wilkinson (Eds.), The international series of monographs on physics (3rd ed.). Oxford, UK: Oxford University Press.

Johnson, S. G. (n.d.). The NLopt nonlinear-optimization package [Computer software]. Retrieved from http://ab-initio.mit.edu/nlopt

Judd, C. H. (1902). Practice and its effects on the perception of illusions. Psychological Review, 9, 27-39.

Kopiske, K. K., Bruno, N., Hesse, C., Schenk, T., \& Franz, V.H. (2016). The functional subdivision of the visual brain: Is there a real illusion effect on action? A multi-lab replication study. Cortex, 79, 130-152.

Kopiske, K. K., Cesanek, E., Campagnoli, C., \& Domini, F. (2017). Adaptation effects in grasping the Müller-Lyer illusion. Vision Research, 136, 21-31.

Krakauer, J. W., Ghez, C., \& Ghilardi, M. F. (2005). Adaptation to visuomotor transformation: Consolidation, interference, and forgetting. The Journal of Neuroscience, 25(2), 473-478.

Lewis, E. O. (1908). The effect of practice on the perception of the Müller-Lyer illusion. British Journal of Psychology, 2, 294-306.

Milner, A. D., \& Goodale, M. A. (2008). Two visual systems re-viewed. Neuropsychologia, 46, 774-785.

Pavani, F., Boscagli, I., Benvenuti, F., Rabuffetti, M., \& Farnè, A. (1999). Are perception and action affected differently by the Titchener circles illusion?. Experimental Brain Research, 127(1), 95-101.

Pine, Z. M., Krakauer, J. W., Gordon, J., \& Ghez, C. (1996). Learning of scaling factors and reference axes for reaching movements. NeuroReport, 7, 2357-2361.

Post, R. B. \& Welch, R. B. (1996). Is there dissociation of perceptual and motor responses to figural illusions? Perception, 25, 569-581.

Powell, M. J. D. (1998). Direct search algorithms for optimization calculations. Acta Numerica, 7, 287-336.

Predebon, J. (2006). Decrement in the Müller-Lyer and Poggendorff illusions: The effects of inspection and practice. Psychological Research, 70, 384-394.

R Core Team. (2014). R: A language and environment for statistical computing [Computer software]. Vienna, Austria: R Foundation for Statistical Computing. Retrieved from http://www.R-project.org/

Säfström, D., \& Edin, B. B. (2008). Prediction of object contact during grasping. Experimental Brain Research, 190(3), 265-277.

Schenk, T. (2012). No dissociation between perception and action in patient DF when haptic feedback is withdrawn. The Journal of Neuroscience, 32(6), 2013-2017.

Schot, W. D., Brenner, E., \& Smeets, J. B. J. (2010). Robust movement segmentation by combining multiple sources of information. Journal of Neuroscience Methods, 187, 147-155.

Smeets, J. B. J., \& Brenner, E. (1999). A new view on grasping. Motor Control, 3, 237-271

Smeets, J. B. J., Glover, S., \& Brenner, E. (2003). Modeling the timedependent effect of the Ebbinghaus illusion on grasping. Spatial Vision, 16(3/4), 311-324.

Stöttinger, E., Aigner, S., Hanstein, K., \& Perner, J. (2009). Grasping the diagonal: Controlling attention to illusory stimuli for action and perception. Consciousness and Cognition, 18, 223-228.

Stöttinger, E., Soder, K., Pfusterschmied, J., Wagner, H., \& Perner, J. (2010). Division of labour within the visual system: Fact or fiction? Which kind of evidence is appropriate to clarify this debate? Experimental Brain Research, 202, 79-88. 
Thoroughman, K. A., \& Shadmehr, R. (2000). Learning of action through adaptive combination of motor primitives. Nature, 407, 742-747.

Verstynen, T., \& Sabes, P. N. (2011). How each movement changes the next: An experimental and theoretical study of fast adaptive priors in reaching. Journal of Neuroscience, 31(27), 10050-10059.

Vishton, P. M., Rea, J. G., Cutting, J. E., \& Nuñez, L. N. (1999). Comparing effects of the horizontal-vertical illusion on grip scaling and judgment: Relative versus absolute, not perception versus action. Journal of Experimental Psychology: Human Perception and Performance, 25(6), 1659-1672.
Voudouris, D., Smeets, J. B. J., \& Brenner, E. (2013). Ultra-fast selection of grasping points. Journal of Neurophysiology, 110, 1484-1489.

Whitwell, R. L., Buckingham, G., Enns, J. T., Chouinard, P. A., \& Goodale, M. A. (2016). Rapid decrement in the effects of the Ponzo display dissociates action and perception. Psychonomic Bulletin \& Review, 23(4), 1157-1163.

Whitwell, R. L., \& Goodale, M. A. (2013). Grasping without vision: Time normalizing grip aperture profiles yields spurious grip scaling to target size. Neuropsychologia, 51(10), 1878-1887. 